\title{
Review Article \\ Theoretical and Phenomenological Status of Neutrino Physics: A Brief Review
}

\author{
Surender Verma \\ Department of Physics and Astronomical Science, Central University of Himachal Pradesh, Dharamshala 176215, India \\ Correspondence should be addressed to Surender Verma; s_7verma@yahoo.co.in
}

Received 16 December 2014; Accepted 11 February 2015

Academic Editor: Elias C. Vagenas

Copyright (C) 2015 Surender Verma. This is an open access article distributed under the Creative Commons Attribution License, which permits unrestricted use, distribution, and reproduction in any medium, provided the original work is properly cited. The publication of this article was funded by SCOAP ${ }^{3}$.

\begin{abstract}
We present an overview of recent progress in the theoretical and phenomenological studies of neutrino masses, lepton avor mixing, and CP violation. Firstly, We discuss the status of neutrino mass with in the Standard Model (SM) of particle physics. Then the possible ways in which neutrino mass terms can be included in the SM are discussed. The inclusion of new physics beyond the SM inevitably brings new parameters which are not constrained by the present experimental data on neutrino masses and mixing angles and, thus, are free parameters of the theory. We, also, discuss various theoretically motivated phenomenological approaches which can be used to reduce the number of free parameters and, thus, provide an excellent tool to understand the underlying physics of neutrino masses and mixings. Current experimental constraints on the neutrino mass spectrum and the lepton avor mixing parameters, including the recent observation of nonzero $\theta_{13}$, have been summarized. Finally, We discuss the renewed interest in the possible existence of one or more sterile neutrinos and their phenomenology.
\end{abstract}

\section{Introduction}

Neutrino physics dates back to early years of the twentieth century when nuclear beta decay experiments $[1,2]$ discovered the continuous spectrum of beta rays which posed a serious problem for theoretical interpretation. There were two possible ways to justify the observed continuous beta ray spectrum: (i) energy conservation does not hold in the nucleus or, alternatively, (ii) a neutral particle that carries missing energy is emitted together with electrons. The second viewpoint was advocated by Pauli in a public letter to "Gruppe der Radioaktiven" at the Gauvereins Tagung in Tubingen written in December 1930 [3] as a "verzweifelter ausweg" (desperate wayout) to save the law of conservation of energy in nuclear beta decay process. According to Pauli's conjecture, the postulated particle should be electrically neutral with a mass much smaller $\left(0.01 m_{p}\right.$ at most $)$ than the proton. The particle was supposed to have spin $1 / 2$ and to respect the Pauli exclusion principle. A pioneering experiment by Ray Davis and his team needed 30 years to catch 2000 solar electron neutrinos in a mine in south Dakota. His count was much lower than predicted by theoretical calculations.
The disappearance of neutrinos seems to be tied to a rather mind boggling behavior of these elusive particles: neutrino oscillations. As neutrinos travel through matter and space, they transform from one type into another, either appearing as electron neutrino, muon neutrino, or tau neutrino. It took almost 26 years for the experimental detection of the neutrinos and, thus, validate the Pauli neutrino hypothesis. The nuclear beta decay experiments, thus, gave us glimpses of the intricate working of nature. Since then, after decades of painstaking experimental and theoretical work, neutrinos have become an essential part of the quantum description of the fundamental particles and forces, namely, the Standard Model (SM) of particle physics.

Neutrino physics has played a crucial role in particle physics since the birth of the theory of weak interaction. The advances in the field requiring the detection of such an elusive particle have been characterized by long time scales until the observation of Super-Kamiokande (SK) experiment in 1998 which triggered an impressive acceleration and interest in the field. Questions about neutrino oscillations and a fourth neutrino are only some of many neutrino puzzles yet to be solved. Exactly how heavy is a single neutrino? What do 
neutrinos tell us about the origin of mass? Are neutrinos connected to extra dimensions? Do neutrinos violate the matterantimatter symmetry or other fundamental symmetries of the Universe? Are neutrinos Dirac or Majorana type?, are some of the puzzles, yet to be answered, in neutrino physics. There are various neutrino experiments primarily aiming to confront these questions. Turning these theoretical ideas and possibilities into reality will require years of research and development to overcome technological challenges nothing new for neutrino physicists, who have seen that the need for patience when unraveling the mysteries of the Universe is their goal. At present, neutrino physics is passing through a phase of spectacular advancements on both theoretical and experimental fronts $[4,5]$. Various neutrino experiments have conclusively demonstrated that neutrinos not only have mass but they also mix and the observed pattern of masses and mixings is quite different from that of the quark sector.

\section{A Bird Eye View of the Standard Model of Particle Physics}

The Standard Model (SM) of particle physics is one of the most successful models in modern physics. It is a mathematical description of interactions of the fundamental building blocks of nature, that is, quarks and leptons. Moreover, it is a quantum description of the three out of four fundamental interactions (SM does not include gravity) mediated by the exchange of particles: the electromagnetic (EM) interaction is mediated by the exchange of photons, the strong interaction by the exchange of gluons, and weak interaction by the exchange of massive vector bosons.

In technical terms, the SM is based on the gauge group

$$
G_{\mathrm{SM}}=S U(3)_{C} \times S U(2)_{L} \times U(1)_{Y}
$$

with three fermion generations. Each generation consists of five different representations of this gauge group:

$$
\left(1,2,-\frac{1}{2}\right),\left(3,2, \frac{1}{6}\right),(1,1,-1),\left(3,1, \frac{2}{3}\right),\left(3,1,-\frac{1}{3}\right),
$$

where the numbers in the parenthesis represent the corresponding charges under the gauge group. In this notation, the electric charge is given by

$$
\mathrm{Q}_{\mathrm{EM}}=T_{L 3}+Y \text {. }
$$

The field and matter content of the SM required to describe the observed elementary particle interactions are given in Tables 1 and 2, respectively. In addition, the SM contains a single Higgs boson doublet $\phi$ with charges $(1,2,1 / 2)$ whose vacuum expectation value (vev)

$$
\langle\phi\rangle=\left(\begin{array}{c}
0 \\
\frac{v}{\sqrt{2}}
\end{array}\right)
$$

breaks the gauge symmetry of the SM

$$
S U(3)_{C} \times S U(2)_{L} \times U(1)_{Y} \longrightarrow S U(3)_{C} \times U(1)_{Y}
$$

TABLE 1: The gauge field content of the SM.

\begin{tabular}{lcc}
\hline & Related symmetry & Fields \\
\hline \multirow{2}{*}{ Electroweak bosons } & $U(1)_{Y}$ & $B_{\mu}$ \\
& $S U(2)_{L}$ & $W_{\mu}^{i}(i=1,2,3)$ \\
\hline Gluons & $S U(3)_{C}$ & $V_{\mu}^{j}(j=1, \ldots, 8)$ \\
\hline
\end{tabular}

This is the only piece of the SM which is not confirmed experimentally. Indeed, the search for the Higgs boson remains one of the premier challenges for the present and future high energy experiments. It can be seen from Table 2 that neutrinos are fermions that have neither strong nor electromagnetic interactions; that is, they are singlets under the symmetry $S U(3)_{C} \times U(1)_{Y}$. The SM has three active neutrinos accompanying the charged leptons $e, \mu$, and $\tau$ and there are weak charged current (CC) interactions between the neutrinos and the corresponding charged leptons given by

$$
-\mathscr{L}_{\mathrm{CC}}=\frac{g}{\sqrt{2}} \sum_{l} \bar{\nu}_{L l} \gamma^{\mu} l_{L}^{-} W_{\mu}^{+}+\text {h.c.. }
$$

In addition, SM neutrinos have, also, neutral current (NC) interactions:

$$
-\mathscr{L}_{\mathrm{NC}}=\frac{g}{2 \cos 2 \theta_{W}} \sum_{l} \bar{\nu}_{L l} \gamma^{\mu} \nu_{L l} Z_{\mu}^{o}
$$

Equations (6) and (7) describe, within the SM, all the neutrino interactions. In the SM, fermion masses arise from the Yukawa interactions which couple a right-handed fermion with its left-handed doublet and the Higgs field in the following manner:

$$
\begin{aligned}
-\mathscr{L}_{\text {Yukawa }}= & Y_{i j}^{d} \bar{Q}_{L i} \phi D_{R j}+Y_{i j}^{u} \bar{Q}_{L i} \tilde{\phi} U_{R j}+Y_{i j}^{l} \bar{l}_{L i} \phi E_{R j} \\
& + \text { h.c. },
\end{aligned}
$$

(where $\tilde{\phi}=i \tau_{2} \phi^{*}$ ) which after spontaneous symmetry breaking lead to charged fermion masses:

$$
m_{i j}^{f}=Y_{i j}^{f} \frac{v}{\sqrt{2}}
$$

In the SM neutrinos are massless. This is due to the fact that, in the SM, neutrinos are described by left-handed chiral fields $v_{e L}, v_{\mu L}$, and $v_{\tau L}$ only. Since corresponding right-handed fields $\nu_{e R}, \nu_{\mu R}$, and $\nu_{\tau R}$ do not exist in the SM, a Dirac mass term

$$
\mathscr{L}^{D}=\sum_{\alpha, \beta=e, \mu, \tau} \bar{v}_{\alpha L} M_{\alpha \beta}^{D} \nu_{\beta R}+\text { h.c. }
$$

is precluded. An important feature of the SM relevant for neutrino mass is the fact that the SM with the gauge 
TABLE 2: Particle content of the SM.

\begin{tabular}{lcccc}
\hline$l_{L}(1,2,-1 / 2)$ & $Q_{L}(3,2,1 / 6)$ & $E_{R}(1,1,-1)$ & $U_{R}(3,1,2 / 3)$ & $D_{R}(3,1,-1 / 3)$ \\
\hline$\left(\begin{array}{c}v_{e} \\
e\end{array}\right)_{L}$ & $\left(\begin{array}{l}u \\
d\end{array}\right)_{L}$ & $e_{R}$ & $u_{R}$ & $d_{R}$ \\
$\left(\begin{array}{c}v_{\mu} \\
\mu\end{array}\right)_{L}$ & $\left(\begin{array}{l}c \\
s\end{array}\right)_{L}$ & $c_{R}$ & $s_{R}$ \\
$\left(\begin{array}{c}v_{\tau} \\
\tau\end{array}\right)_{L}$ & $\left(\begin{array}{l}t \\
b\end{array}\right)_{L}$ & $\mu_{R}$ & $t_{R}$ & $b_{R}$ \\
\hline
\end{tabular}

symmetry (equation (1)) and the particle content of Table 2 has an accidental global symmetry

$$
G_{\mathrm{SM}}^{\text {global }}=U(1)_{B} \times U(1)_{L_{e}} \times U(1)_{L_{\mu}} \times U(1)_{L_{\tau}},
$$

where $U(1)_{B}$ is the baryon number symmetry and $U(1)_{L_{e}, L_{\mu}, L_{\tau}}$ are the three lepton flavor symmetries with total lepton number given by $L=L_{e}+L_{\mu}+L_{\tau}$. It is an accidental symmetry in the sense that we do not impose it. It is a consequence of the gauge symmetry and the representations of the physical states. In principle, neutrino mass can arise from loop corrections. In the SM, however, this cannot happen because the only possible neutrino mass term that can be constructed with the SM fields is the bilinear $l_{L} l_{L}^{C}$ which violates the total lepton number symmetry by two units. But as mentioned above, total lepton number is a global symmetry of the SM and therefore $L$-violating terms cannot be induced by loop corrections. Thus, the field content and the gauge symmetries of the SM preclude the existence of neutrino mass. This prediction of the SM is in contradiction with the experimental evidence for neutrino masses and mixings [6-12]. Therefore, it is necessary to extend the SM to accommodate nonzero neutrino masses.

The simplest possible extension of the SM is the introduction of the three right-handed neutrino fields $\nu_{e R}, v_{\mu R}$, and $\nu_{\tau R}$ which are singlets under the gauge symmetry of the SM. In this way the neutrino fields become similar to the other massive fermion fields which have both left-handed and right-handed components. The Dirac mass term for the neutrino fields can be generated in a manner similar to Dirac masses for charged leptons and quarks. In addition, the Majorana mass term for the right-handed neutrinos can be written as

$$
\mathscr{L}_{R}^{M}=\frac{1}{2} \sum_{\alpha, \beta=e, \mu, \tau} \overline{v_{\alpha L}^{C}}\left(M_{R}^{M}\right)_{\alpha \beta} v_{\beta R}+\text { h.c. }
$$

which is invariant under the gauge symmetries of the SM and is, hence, allowed. The fundamental difference between a Majorana fermion and a Dirac fermion is that, for a Majorana fermion, the particle and antiparticle states coincide. In other words, charge conjugation does not have any effect on a Majorana fermion field. So $\left(\nu_{\alpha L}\right)^{C}=\nu_{\alpha R}^{C}$ for $\alpha=e, \mu, \tau$. The introduction of right-handed neutrino fields leads to new physics, that is, Majorana neutrino masses and the existence of processes which violate lepton number by two units $(|\Delta L|=2)$. In general, in a model with left-handed and right-handed neutrino fields, the neutrino mass term is given by

$$
\begin{aligned}
\mathscr{L}^{D+M} & =\mathscr{L}^{D}+\mathscr{L}_{R}^{M} \\
& =\frac{1}{2}\left(\begin{array}{ll}
\bar{\nu}_{L} & \overline{\nu_{L}^{C}}
\end{array}\right)\left(\begin{array}{cc}
0 & M^{D} \\
\left(M^{D}\right)^{T} & M_{R}^{M}
\end{array}\right)\left(\begin{array}{l}
\nu_{R}^{C} \\
\nu_{R}
\end{array}\right)+\text { h.c. } .
\end{aligned}
$$

The Dirac mass matrix $M^{D}$ is generated by the Higgs mechanism of the SM and its elements are proportional to the VEV of the Higgs doublet $v_{\mathrm{SM}}=\left(\sqrt{2} G_{F}\right)^{-1 / 2}=246 \mathrm{GeV}$, where $G_{F}$ is the Fermi constant. Thus, the magnitudes of the elements of $M^{D}$ are protected by the gauge symmetries of the SM. On the other hand, the Majorana mass term for the neutrino fields is invariant under the gauge symmetries of the SM; the elements of $M_{R}^{M}$ are not protected by the SM gauge symmetries. In other words, the elements of $M_{R}^{M}$ can have arbitrary large values. If $M_{R}^{M}$ is generated by the Higgs mechanism at a high energy scale of new physics beyond the $\mathrm{SM}$, the elements of $M_{R}^{M}$ are expected to be of the order of such high energy scale which could be as high as the grand unification scale of about $10^{15} \mathrm{GeV}$. For $M_{R}^{M} \gg M^{D}$ the neutrino mass matrix

$$
M=\left(\begin{array}{cc}
0 & M^{D} \\
\left(M^{D}\right)^{T} & M_{R}^{M}
\end{array}\right)
$$

can be diagonalized to yield the effective light Majorana neutrino mass matrix given by

$$
m_{\mathrm{eff}}=-M^{D}\left(M_{R}^{M}\right)^{-1}\left(M^{D}\right)^{T}
$$

and a heavy $3 \times 3$ mass matrix

$$
M_{\text {heavy }} \approx M_{R}^{M} .
$$

Therefore, there are three heavy neutrinos decoupled from the low energy physics and three light neutrinos whose masses are suppressed with respect to the elements of Dirac mass matrix $M^{D}$ by the small matrix factor $\left(M_{R}^{M}\right)^{-1}\left(M^{D}\right)^{T}$ which is known as seesaw mechanism [13-16]. 


\section{The General Structure of Lepton Mixing Matrix}

In any gauge theory, in order to identify physical particles, one must diagonalize all relevant mass matrices which typically result from gauge symmetry breaking. Mechanisms giving mass to neutrinos generally imply the need for new interactions whose Yukawa couplings will coexist with that of the charged leptons. The lepton mixing matrix, $U$, follows from a mismatch between the diagonalization of the charged lepton mass matrix and that of the neutrino mass matrix. This is similar to the way the Cabibbo-Kobayashi-Maskawa (CKM) matrix arises in the quark sector from a mismatch between up- and down-type Yukawa couplings. Hence, like quarks, massive neutrinos will generally mix. The means that the flavor states $\nu_{\alpha}, \alpha=e, \mu, \tau$ do not coincide with the mass eigenstates $v_{i}, i=1,2,3$. The flavor states are combinations of the mass eigenstates

$$
\nu_{\alpha}=U_{\alpha i} \nu_{i}
$$

where the mixing parameters $U_{\alpha i}$ form the PMNS mixing matrix, $U_{\mathrm{PMNS}}$. In the mass basis, leptonic charged current interaction is given by

$$
-\mathscr{L}_{\mathrm{CC}}=\frac{g}{\sqrt{2}}\left(\begin{array}{lll}
\bar{e}_{L} & \bar{\mu}_{L} & \bar{\tau}_{L}
\end{array}\right) \gamma^{\mu} U\left(\begin{array}{c}
\nu_{1} \\
v_{2} \\
\nu_{3}
\end{array}\right) W_{\mu}^{-}+\text {h.c. }
$$

where $\left(\nu_{1}, \nu_{2}, v_{3}\right)$ and $(e, \mu, \tau)$ are neutrino mass eigenstates and charged lepton mass eigenstates, respectively. $U$ is a $3 \times 3$ unitary lepton mixing matrix with $U U^{\dagger}=1$. The mass term is a sum of Lorentz invariant products of left-handed and righthanded components of the fields. The charge conjugate fields $\nu_{L}^{C}=C \bar{\nu}_{L}^{T}$ and $\nu_{R}^{C}=C \bar{\nu}_{R}^{T}$ are right-handed and left-handed, respectively. Here, $C$ is the unitary charge conjugation matrix having $C \gamma_{\mu}^{T} C^{-1}=-\gamma_{\mu}, C^{T}=-C$. Using these we can find that $\overline{\nu_{L}^{C}}=-v_{L}^{T} C^{-1}$ and $\overline{\nu_{R}^{C}}=-v_{R}^{T} C^{-1}$. Taking into account that $v_{L}^{C}$ is the right-handed component one can easily write the mass term for neutrinos. In the basis where both charged lepton and the neutrino mass matrices are nondiagonal, the mass term for the leptons can be written as

$$
\mathscr{L}_{m}=\left[v_{L}^{T} C^{-1} M_{\nu} \nu_{L}+\overline{l_{L}} M_{l} l_{R}+\text { h.c. }\right] .
$$

The charged lepton mass matrix " $M_{l}$ " can be diagonalized by the matrices $V^{l}$ and $V_{R}^{l}$ :

$$
V^{l \dagger} M_{l} V_{R}^{l}=\left(\begin{array}{ccc}
m_{e} & 0 & 0 \\
0 & m_{\mu} & 0 \\
0 & 0 & m_{\tau}
\end{array}\right) \text {. }
$$

The complex symmetric Majorana neutrino mass matrix $M_{v}$ can be diagonalized by an orthogonal matrix $V^{\nu}$ :

$$
V^{v} M_{\nu} V^{\nu T}=\left(\begin{array}{ccc}
m_{v_{e}} & 0 & 0 \\
0 & m_{v_{\mu}} & 0 \\
0 & 0 & m_{v_{\tau}}
\end{array}\right) .
$$

The lepton mixing matrix $U_{\mathrm{PMNS}}$ is given by

$$
\left(U_{\mathrm{PMNS}}\right)_{i j}=V_{i k}^{l \dagger} V_{k j}^{v}
$$

For Majorana (Dirac) neutrinos $U_{\text {PMNS }}$ contains (for $n$ generations) a total of $6(n-2)[5 n-11]$ real parameters out of which $3(n-2)$ are angles and $3(n-2)[2 n-5]$ can be interpreted as physical phases. In particular, if there are only three Majorana neutrinos, $U_{\mathrm{PMNS}}$ is a $3 \times 3$ unitary matrix analogous to the CKM matrix for quarks [17] and depends upon six real parameters which include three mixing angles and three phases. It can be conveniently parameterized as

$$
\begin{aligned}
U_{\mathrm{PMNS}} & \left(\begin{array}{ccc}
c_{12} c_{13} & s_{12} c_{13} & s_{13} e^{-i \delta} \\
-s_{12} c_{23}-c_{12} s_{13} s_{23} e^{i \delta} & c_{12} c_{23}-s_{12} s_{13} s_{23} e^{i \delta} & c_{13} s_{23} \\
s_{12} s_{23}-c_{12} s_{13} c_{23} e^{i \delta} & -c_{12} s_{23}-s_{12} s_{13} c_{23} e^{i \delta} & c_{13} c_{23}
\end{array}\right) \\
& \cdot\left(\begin{array}{ccc}
1 & 0 & 0 \\
0 & e^{i \alpha} & 0 \\
0 & 0 & e^{i(\beta+\delta)}
\end{array}\right),
\end{aligned}
$$

where $c_{i j} \equiv \cos \theta_{i j}$ and $s_{i j}=\sin \theta_{i j}$. The phases $\delta$ and $(\alpha$, $\beta$ ) are Dirac-type and Majorana-type $C P$ violating phases, respectively.

\section{Neutrino Oscillations}

Neutrino oscillations are a manifestation of leptonic mixing. The first hint that neutrinos have mass came from the pioneering Homestake experiment [18] performed by the American scientist Raymond Davis Jr. for the detection of solar neutrinos. In this experiment, it was found that only about one-third of the number of neutrinos predicted by standard solar model (SSM) were reaching the detector on the earth. This result puzzled both the solar and neutrino physicists. However, Mikheyev and Smirnov [19] put forward the idea, proposed previously by Wolfenstein [20], that the solar neutrinos might be changing into something else. Only electron neutrinos are emitted by the Sun and they could be converting into muon and tau neutrinos which were not being detected by the experiment. This effect of neutrino interconversion over time from one kind to another, namely, "neutrino oscillations," was first proposed by Gribov and Pontecorvo [21]. The precise mechanism for "solar neutrino oscillations" proposed by Mikheyev, Smirnov, and Wolfenstein (MSW) involved the resonant enhancement of neutrino oscillations due to matter effects. Just as light passing through the matter slows down, which is equivalent to the photon gaining a small effective mass, neutrinos passing through matter also get slowed down and gain a small effective mass. The effective neutrino mass is largest when matter density is highest which in the case of solar neutrinos is in the core of the Sun. In particular, electron neutrinos generated in the core of the Sun will be subject to such matter effects. 
This can result in a resonant enhancement of solar neutrino oscillations which is known as the MSW effect [20].

The idea of neutrino oscillations gained support from the Japanese experiment Super-Kamiokande [22, 23] in which similar deficit was observed in the atmospheric neutrino flux. The atmospheric neutrinos are produced when cosmic rays, composed mainly of protons, interact with the upper atmosphere. Most of the neutrinos pass through the earth unhindered; Super-Kamiokande was able to detect muon neutrinos coming from above as well as below and found that while the expected number of muons was coming from above only about a half of the expected number came from below. These results were attributed to the muon neutrinos coming from below having oscillated into tau neutrinos over an oscillation length $L$ equal to the diameter of the earth. However, a number of muon neutrinos coming from above have negligible oscillation length and have no time to oscillate yielding the expected number of muon neutrinos from above. More recently, the Sudbury Neutrino Observatory (SNO) [24, 25] in Canada has confirmed the solar neutrino oscillations. There are various other neutrino experiments which not only have confirmed the neutrino oscillation phenomenon but also have measured the neutrino oscillation parameters with increased accuracies [26]. In fact, through neutrino oscillations, quantum mechanics allowed us to probe the smallest masses measured so far. More importantly, the results of neutrino experiments are, thus, providing the motivation for probing physics beyond the SM.

4.1. Neutrino Oscillations in Vacuum. Neutrino oscillations are quantum mechanical phenomenon in which a neutrino born with flavor $\alpha$ changes to a different flavor $\beta$ while propagating in vacuum or in matter. Observation of neutrino oscillations in various neutrino experiments has shown that there is a mismatch between the flavor and mass eigenstates of neutrinos. Neutrinos are produced and detected via weak interactions. The neutrino state created in the decay $W^{+} \rightarrow l_{\alpha}^{+}+v$ is given by

$$
\left|\nu_{\alpha}\right\rangle=\sum_{i=1}^{n} U_{\alpha i}^{*}\left|\nu_{i}\right\rangle
$$

This superposition of neutrino mass eigenstates produced in association with the charged lepton of flavor $\alpha$ is the state we refer to as the neutrino of flavor $\alpha$. It is evident from (24) that the probability of finding a neutrino created in a given flavor to be in the same state (or any other state) oscillates with time. The time evolution of a neutrino produced in a given flavor at $x=t=0$ is given by

$$
\left|\nu_{\alpha}(t)\right\rangle=\sum_{i=1}^{n} U_{\alpha i}^{*}\left|\nu_{i}(t)\right\rangle=\sum_{i=1}^{n} U_{\alpha i}^{*} e^{-i E_{i} t}\left|\nu_{i}(0)\right\rangle .
$$

The neutrino oscillation probability that is, the probability of transformation from one flavor eigenstate $\left|\nu_{\alpha}\right\rangle$ to another flavor eigenstate $\left|\nu_{\beta}\right\rangle$ is given by

$$
\begin{aligned}
P\left(v_{\alpha} \longrightarrow v_{\beta}\right) & =P_{\alpha \beta}=\left|\left\langle v_{\beta} \mid v_{\alpha}(t)\right\rangle\right|^{2} \\
& \left.=\left|\sum_{i=1}^{n} \sum_{j=1}^{n} U_{\alpha i}^{*} U_{\beta j}\right| v_{j}\right\rangle\left. v_{i}(t)\right|^{2} .
\end{aligned}
$$

For ultrarelativistic neutrinos, one can assume $p_{i} \simeq p_{j} \equiv p=$ E so that

$$
E_{i}=\sqrt{p_{i}^{2}+m_{i}^{2}} \simeq p+\frac{m_{i}^{2}}{2 E}
$$

where $E_{i}$ and $m_{i}$ are the energy and mass of the neutrino mass eigenstate $v_{i}$. Using (27) along with the orthogonality relation $\left\langle v_{j} \mid v_{i}\right\rangle=\delta_{i j}$ we obtain the transition probability $P_{\alpha \beta}$ given by

$$
\begin{aligned}
P_{\alpha \beta}= & \delta_{\alpha \beta}-4 \sum_{i>j} \Re\left(U_{\alpha i}^{*} U_{\beta i} U_{\alpha j} U_{\beta j}^{*}\right) \sin ^{2}\left[1.27 \Delta m_{i j}^{2}\left(\frac{L}{E}\right)\right] \\
& +2 \sum_{i>j} \Im\left(U_{\alpha i}^{*} U_{\beta i} U_{\alpha j} U_{\beta j}^{*}\right) \sin \left[2.54 \Delta m_{i j}^{2}\left(\frac{L}{E}\right)\right],
\end{aligned}
$$

where $\Delta m_{i j}^{2} \equiv m_{i}^{2}-m_{j}^{2}$ is in $\mathrm{eV}^{2}, L$ is in $\mathrm{km}$, and $E$ is in $\mathrm{GeV}$. Thus, the essential ingredients for neutrino oscillations to occur are twofold: one is that neutrinos must not only be massive but also nondegenerate in mass and the second is that mass eigenstates of the neutrinos must be different from the flavor eigenstates. Assuming that CPT invariance holds

$$
P\left(\bar{\nu}_{\alpha} \longrightarrow \bar{v}_{\beta}\right)=P\left(\nu_{\beta} \longrightarrow v_{\alpha}\right)
$$

But from (28), we see that

$$
P\left(v_{\beta} \longrightarrow v_{\alpha} ; U\right)=P\left(v_{\alpha} \longrightarrow v_{\beta} ; U^{*}\right)
$$

Thus when CPT holds,

$$
P\left(\bar{v}_{\alpha} \longrightarrow \bar{v}_{\beta} ; U\right)=P\left(v_{\alpha} \longrightarrow v_{\beta} ; U^{*}\right)
$$

that is, the probability for oscillation of an antineutrino is the same as that for a neutrino, but the mixing matrix $U$ is replaced by its complex conjugate. Thus, if $U$ is not real, the neutrino and antineutrino oscillation probabilities can differ by having opposite sign in the last term in (28). When CPT holds any difference in these probabilities, this will indicate violation of $C P$ invariance. For the case of two generations of neutrinos, the above analysis becomes quite simplified without any loss of physical understanding. The mixing matrix depends only on one parameter $\theta$ (known as mixing angle) and is given by

$$
U=\left(\begin{array}{cc}
\cos \theta & \sin \theta \\
-\sin \theta & \cos \theta
\end{array}\right)
$$

and there is a single mass-squared difference $\Delta m^{2}$. With this form of $U$ (28) becomes

$$
P_{\alpha \beta}=\sin ^{2} 2 \theta \sin ^{2}\left(1.27 \Delta m^{2} \frac{L}{E}\right) .
$$


4.2. Neutrino Oscillations in Matter. The propagation of neutrinos becomes significantly modified in the presence of matter due to their interactions with the matter composed of electrons, protons, and neutrons. Moreover, the coherent forward elastic scattering amplitudes are not the same for all neutrino flavors, that is, $v_{e}, v_{\mu}$, and $\nu_{\tau}$. The electron neutrinos $\nu_{e}$ have additional contribution due to their charged current (CC) interactions with matter which are mediated by $W^{ \pm}$ bosons. On the basis of this fact, Mikheyev and Smirnov [19], following the work of Wolfenstein [20], showed that an exciting phenomenon occurs when neutrinos travel through dense matter. In the two-flavor case, if neutrinos $\nu_{e}$ and $\nu_{\mu}$ travel through the Sun, the propagation of $\nu_{1}$ and $v_{2}$ will be modified due to the different interactions of $\nu_{e}$ and $\nu_{\mu}$ with electrons. The mixing angle in the presence of matter is given by

$$
\sin ^{2} 2 \theta_{m}=\frac{\sin ^{2} 2 \theta}{\left(\cos 2 \theta-L / L_{m}\right)^{2}+\sin ^{2} 2 \theta},
$$

where $L_{m}$, a length characteristic of motion in matter of density $N_{e}$, is given by

$$
L_{m}=\frac{2 \pi}{\sqrt{2} G_{F} N_{e}},
$$

where $G_{F}$ is the Fermi constant. Mikheyev and Smirnov [19] pointed out that (34) has a resonant character. If the neutrinos travel through a varying matter density, one may have

$$
\frac{L}{L_{m}}=\cos 2 \theta
$$

so that

$$
\sin ^{2} 2 \theta_{m}=1
$$

resulting in maximal mixing and the survival probability [27], to a very good approximation, can be written as

$$
P_{\alpha \alpha}=\frac{1}{2}+\frac{1}{2} \cos 2 \theta \cos 2 \theta_{m}
$$

\section{Recent Developments in Neutrino Phenomenology and the Current Status of Neutrino Masses and Mixings}

Spectacular advancements on both the theoretical and the experimental fronts have made possible the measurements of neutrino oscillation parameters. Recent major progress in neutrino physics, in particular and particle physics, in general, is related to studies of the neutrino masses and mixings. The first phase of these studies is essentially over and the main results from this phase are summarized in the following:

(1) discovery of nonzero neutrino mass;

(2) determination of the dominant structure of the lepton mixing, that is, discovery of two large (solar mixing angle $\theta_{12}$ and atmospheric mixing angle $\theta_{23}$ ) and one small (reactor) mixing angles;

(3) establishing strong differences in the quark and lepton mass spectra and their mixing patterns.
In a sense, we have now a "standard model of neutrinos" that can be formulated in the following way:

(1) there are only three types of light neutrinos;

(2) their interactions are described by the standard electroweak theory;

(3) masses and mixings are generated in vacuum and they originate from some high energy (short range) physics at the electroweak and/or higher scales.

However, there exist many open questions about massive neutrinos and lepton flavor mixing. The following are examples.

(1) Are massive neutrinos Dirac or Majorana particles? If massive neutrinos are Dirac particles, they can be distinguished from their antiparticles. By definition, a Majorana neutrino is identical to its antiparticle. It is possible to identify the Majorana nature of massive neutrinos through the observation of the neutrinoless double beta decay of some even-even nuclei in which the total lepton number is not conserved.

(2) Why are neutrino masses so tiny? The fact that the masses of neutrinos are considerably smaller than the masses of charged leptons or quarks is a big puzzle in particle physics. Although, a lot of theoretical models about neutrino masses have been proposed at either low or high energy scales, none of them has proved to be very successful and conceivable.

(3) What is the absolute scale of neutrino masses? It is very important to know the absolute values of three neutrino masses because they are fundamental parameters of flavor physics. The mass scale of neutrinos is likely to indicate the energy scale of new physics responsible for the generation of neutrino masses and lepton flavor mixing.

(4) Why are the mixing angles $\theta_{12}$ and $\theta_{23}$ so large while $\theta_{13}$ is, relatively, small? The bilarge neutrino mixing pattern is, also, a mystery to theorists because it is "anomalously" different from the familiar trismall quark mixing pattern.

(5) Is there leptonic $C P$ violation? A necessary condition for the existence of $C P$ and $T$ violation in normal neutrino oscillations is $\theta_{13} \neq 0$. As $C P$ violation has been discovered in the quark sector there is no reason why $C P$ should be conserved in the lepton sector.

(6) Can the leptonic $C P$ violating phases be determined? If lepton flavor mixing is correlated with $C P$ violation, one has to determine the relevant $C P$ violating phases through various possible experiments. The neutrinoless double beta decay and long-baseline appearance neutrino oscillations are expected to be sensitive to the Majorana and Dirac-type $C P$ violating phases, respectively.

The immediate goal is to search for new physics beyond the Standard Model. The next phase of studies will be associated with new generation of neutrino experiments. The main 
TABLE 3: Global analysis of solar, atmospheric, reactor, and accelerator neutrino data in the framework of three-neutrino oscillations. Here, $\Delta m_{3 l}^{2} \equiv \Delta m_{31}^{2}$ for $\mathrm{NH}$ and $\Delta m_{3 l}^{2} \equiv \Delta m_{32}^{2}$ for $\mathrm{IH}$.

\begin{tabular}{|c|c|c|c|c|}
\hline \multirow{2}{*}{ Parameter } & \multicolumn{2}{|c|}{ Normal hierarchy $(\mathrm{NH})$} & \multicolumn{2}{|c|}{ Inverted hierarchy (IH) } \\
\hline & Best fit $\pm 1 \sigma$ & $3 \sigma$ range & Best fit $\pm 1 \sigma$ & $3 \sigma$ range \\
\hline $\sin ^{2} \theta_{12}$ & $0.304_{-0.012}^{+0.013}$ & $0.270-0.344$ & $0.304_{-0.012}^{+0.013}$ & $0.270-0.344$ \\
\hline $\sin ^{2} \theta_{23}$ & $0.452_{-0.028}^{+0.052}$ & $0.382-0.643$ & $0.579_{-0.037}^{+0.025}$ & $0.389-0.644$ \\
\hline $\begin{array}{c}\sin ^{2} \theta_{13} \\
\Delta m_{21}^{2}\end{array}$ & $0.0218_{-0.0010}^{+0.0010}$ & $0.0186-0.0250$ & $0.0218_{-0.0010}^{+0.0010}$ & $0.0188-0.0251$ \\
\hline $\begin{array}{c}\frac{21}{10^{-5} \mathrm{eV}^{2}} \\
\Delta m_{3 l}^{2}\end{array}$ & $7.50_{-0.17}^{+0.19}$ & $7.02-8.09$ & $7.50_{-0.17}^{+0.19}$ & $7.02-8.09$ \\
\hline$\overline{10^{-3} \mathrm{eV}^{2}}$ & $+2.457_{-0.047}^{+0.047}$ & $+2.317-+2.607$ & $-2.449_{-0.047}^{+0.048}$ & $-2.590--2.307$ \\
\hline
\end{tabular}

objectives of this new phase include the determination of the absolute scale of neutrino mass and subdominant structures of mixing matrix, namely, deviation of the $\theta_{23}$ from maximal value and the $C P$ violation phase(s). The objectives, also, include identification of neutrino mass hierarchy and precision measurements of already known parameters. For the case of three generations, there are four mixing parameters, namely, the three mixing angles and the Dirac-type phase which will manifest in the neutrino oscillation experiments. The neutrino oscillation experiments are sensitive to only two mass squared differences, all the three mixing angles, and the Dirac-type $C P$ phase. We, already, have fairly good knowledge of the two mass squared differences and all the three mixing angles. Results from solar neutrino experiments [28-32] which have been collecting data for more than four decades have now culminated in choosing the large mixing angle (LMA) solution as a solution of the solar neutrino problem. The latest addition to this huge repertoire of experimental data are the results from the Borexino experiment [33] which are consistent with the LMA solution. This conclusion from the solar neutrino experiments has been investigated independently by the KamLAND reactor antineutrino experiment $[26,34-36]$ and a combined analysis of the solar and KamLAND data gives $\Delta m_{21}^{2}=7.6 \times$ $10^{-5} \mathrm{eV}^{2}$ and $\sin ^{2} 2 \theta_{12}=0.32[12,36-41]$. The other masssquared difference $\Delta m_{32}^{2}$ and mixing angle $\theta_{23}$ are now pretty well determined by the zenith angle dependent atmospheric $\nu_{\mu}$ data from Super-Kamiokande $[22,23,32,42]$ and the long baseline experiments $\mathrm{K} 2 \mathrm{~K}[43,44]$ and MINOS [45]. The combined data from the atmospheric and long baseline experiments have pinned down $\left|\Delta m_{32}^{2}\right|=2.4 \times 10^{-3} \mathrm{eV}^{2}$ and $\sin ^{2} 2 \theta_{23}=1$. With the observation of nonzero $\theta_{13}$ [46-53], we now have complete knowledge of all the three mixing angles $\theta_{12}, \theta_{23}$, and $\theta_{13}$. However, we do not have any information on Dirac-type $C P$ violating phase $\delta$ and it is fully unconstrained as yet. In Table 3, we have shown the present status of the neutrino oscillation parameters [54].

\section{Neutrino Physics and $C P$ Violation}

Symmetries play an important role in physics since they describe the invariance of physical phenomena under fundamental transformations. The symmetries $P, C$, and $T$ correspond to the invariance of the Lagrangian of the field theory under these discrete operations. Parity $(P)$ and time reversal $(T)$ are space-time symmetries. The parity operation reverses all spatial coordinates $(r \rightarrow-r)$ and, in consequence, the directions of all momentum vectors are reversed while leaving spins and angular momenta unchanged. The time reversal operation $T$ corresponds to the replacement of $T$ by $-T$ so that the directions of momenta and spins are reversed. All complex numbers such as coupling constants are replaced by their complex conjugates under the $T$ operation. The operation of charge conjugation " $C$ " is to interchange the role of the annihilation and creation operators of the particle with those of the antiparticle. The $C$ operation flips the signs of the internal charges such as baryon number, electric charge, strangeness, and lepton number.

After observation of parity violation in weak interactions in 1956, the discovery of $C P$ violation in the decay of neutral kaons in 1964 was the major breakthrough in our understanding of the real world. Since then considerable experimental and theoretical work has been devoted to understanding its occurrence and origin. In the SM, the possibility of $C P$ violation arises from the presence, in the Lagrangian, of complex Yukawa couplings between fermions and the Higgs boson. In the quark sector, weak couplings are described by the Cabibbo-Kobayashi-Maskawa (CKM) mixing matrix $[17,55]$ given by

$$
\begin{aligned}
& V_{\text {СКM }} \\
& \quad=\left(\begin{array}{ccc}
c_{12}^{q} c_{13}^{q} & s_{12}^{q} c_{13}^{q} & s_{13}^{q} e^{-i \delta^{q}} \\
-s_{12}^{q} c_{23}^{q}-c_{12}^{q} s_{23}^{q} s_{13}^{q} e^{i \delta^{q}} & c_{12}^{q} c_{23}^{q}-s_{12}^{q} s_{23}^{q} s_{13}^{q} e^{i \delta^{q}} & s_{23}^{q} c_{13}^{q} \\
s_{12}^{q} s_{23}^{q}-c_{12}^{q} c_{23}^{q} s_{13}^{q} e^{i \delta^{q}} & -c_{12}^{q} s_{23}^{q}-s_{12}^{q} c_{23}^{q} s_{13}^{q} e^{i \delta^{q}} & c_{23}^{q} c_{13}^{q}
\end{array}\right),
\end{aligned}
$$

where $c_{i j}^{q}=\cos \theta_{i j}^{q}, s_{i j}^{q}=\sin \theta_{i j}^{q}, \theta_{i j}^{q}$ are quark mixing angles and $\delta^{q}$ is Dirac-type $C P$ violating phase.

Neutrinos were introduced into the SM as massless fermions for which no gauge invariant renormalizable mass term can be constructed. Therefore, there is neither mixing nor $C P$ violation in the leptonic sector and $\delta^{q}$ is the only source of $C P$ violation in the SM. Moreover, the amount of $C P$ violation predicted by the SM is far too small to yield the observed ratio of baryons to photons in the Universe [56-58]. The experimental evidence for neutrino masses and mixings provided an unambiguous signal of new physics where new 
sources of $C P$ violation can be investigated. In the physical basis the mass terms and the leptonic charged currents are given by

$$
\begin{aligned}
L^{\mathrm{phys}}= & \nu_{L}^{T} C d \nu_{L}+\bar{l}_{L} d_{l} l_{R}+\frac{g}{\sqrt{2}} \bar{l}_{i L} \gamma^{\mu}\left(U_{i j}\right)_{\mathrm{PMNS}} \nu_{j L} W_{\mu}^{-} \\
& + \text {h.c., }
\end{aligned}
$$

where $d$ and $d_{l}$ are diagonal, real, and positive matrices. The $3 \times 3$ unitary matrix $U_{\text {PMNS }}$ is, in general, parameterized by six phases and three mixing angles. Three of these phases can be factored out and rotated away through the redefinition of the charged leptons $l_{L}$. These phases, thus, appearing in $d_{l}$ can be eliminated by the simultaneous redefinition of fields $l_{R}$. Another two of the six phases of $U$ can be factored out to the right; however, in this case these two phases are physical because rotating them away from $U$ corresponds to transferring them to the mass term of the light neutrinos. This is an important difference from the quark sector resulting from the fact that in the seesaw framework neutrinos have Majorana masses and are Majorana particles unlike quarks. These factorizable phases which cannot be removed from the theory are called Majorana phases. As a result, in the seesaw framework, there are additional sources of $C P$ violation beyond the Kobayashi Maskawa mechanism of the quark sector. In the leptonic sector, there is one Dirac-type $C P$ violating phase $\delta$ and two Majorana-type $C P$ violating phases $\alpha, \beta$. The PMNS matrix can be parameterized as

$$
\begin{aligned}
U_{\mathrm{PMNS}} & \left(\begin{array}{ccc}
c_{12} c_{13} & s_{12} c_{13} & s_{13} e^{-i \delta} \\
-s_{12} c_{23}-c_{12} s_{13} s_{23} e^{i \delta} & c_{12} c_{23}-s_{12} s_{13} s_{23} e^{i \delta} & c_{13} s_{23} \\
s_{12} s_{23}-c_{12} s_{13} c_{23} e^{i \delta} & -c_{12} s_{23}-s_{12} s_{13} c_{23} e^{i \delta} & c_{13} c_{23}
\end{array}\right) \\
& \cdot\left(\begin{array}{ccc}
1 & 0 & 0 \\
0 & e^{i \alpha} & 0 \\
0 & 0 & e^{i(\beta+\delta)}
\end{array}\right),
\end{aligned}
$$

where $c_{i j}=\cos \theta_{i j}, s_{i j}=\sin \theta_{i j}, \theta_{i j}$ are neutrino mixing angles. Leptonic $C P$ violation at low energies can be observed through neutrino oscillations which are sensitive to the Dirac-type $C P$ phase $\delta$ but insensitive to the Majorana-type $C P$ violating phases. The Dirac-type $C P$ violation can be related to the low energy weak basis (WB) invariant [59]

$$
I_{1}=\operatorname{Tr}\left[H_{v}, H_{l}\right]^{3} \text {, }
$$

where $H_{v}=M_{v} M_{v}^{\dagger}$ and $H_{l}=M_{l} M_{l}^{\dagger}$ with $M_{v}$ and $M_{l}$ being neutrino and charged lepton mass matrices, respectively. In the basis where charged lepton mass matrix is diagonal $I_{1}$ can be written as

$$
\begin{aligned}
I_{1}= & -6 i\left(m_{\mu}^{2}-m_{e}^{2}\right)\left(m_{\tau}^{2}-m_{\mu}^{2}\right)\left(m_{\tau}^{2}-m_{e}^{2}\right) \\
\cdot & \mathfrak{J}\left[\left(H_{\nu}\right)_{12}\left(H_{\nu}\right)_{23}\left(H_{\nu}\right)_{31}\right] .
\end{aligned}
$$

In terms of neutrino masses and mixings, $I_{1}$ is given by

$$
\begin{aligned}
I_{1}= & 6 i\left(m_{\mu}^{2}-m_{e}^{2}\right)\left(m_{\tau}^{2}-m_{\mu}^{2}\right)\left(m_{\tau}^{2}-m_{e}^{2}\right)\left(m_{3}^{2}-m_{1}^{2}\right) \\
& \cdot\left(m_{2}^{2}-m_{1}^{2}\right)\left(m_{3}^{2}-m_{2}^{2}\right) J_{C P},
\end{aligned}
$$

where $J_{C P}=\mathfrak{\Im}\left[U_{11} U_{22} U_{12}^{*} U_{21}^{*}\right]$ determines the magnitude of $C P$ violation in neutrino oscillations. Using (42)

$$
J_{C P}=\frac{1}{8} \sin 2 \theta_{12} \sin 2 \theta_{13} \sin 2 \theta_{23} \cos \theta_{13} \sin \delta .
$$

Thus, a nonzero value of $\theta_{13}$ is an encouraging sign for the prospects of measuring low energy leptonic $C P$ violation mediated through a Dirac-type $C P$ violating phase. In the quark sector, $J_{C P}$ is of the order of $10^{-5}$ [60] whereas in the leptonic sector, with $\theta_{13}$ equal to the present experimental value, $J_{C P}$ can be of the order of $10^{-2}$ which is about three orders of magnitude larger than that in the quark sector. Apart from the WB invariant $I_{1}$ there are two more WB invariants $I_{2}, I_{3}$ [61] related to the Majorana nature of massive neutrinos which are sensitive to the Majorana-type $C P$ violating phases $\alpha, \beta$ and are given by

$$
\begin{aligned}
& I_{2}=\mathfrak{I} \operatorname{Tr}\left[H_{l} M_{v} M_{\nu}^{*} M_{v} H_{l}^{*} M_{\nu}^{*}\right], \\
& I_{3}=\mathfrak{\Im} \operatorname{Det}\left[M_{v}^{*} H_{l} M_{v}, H_{l}^{*}\right] .
\end{aligned}
$$

A necessary and sufficient condition for low energy $C P$ invariance in the leptonic sector is that these three $\mathrm{WB}$ invariants should be identically zero $[62,63]$.

\section{Phenomenology of Neutrino Mass Matrix}

In view of the recent experimental and theoretical developments in neutrino physics, it is quite clear that there exists physics beyond the SM. Almost every extension of the SM has additional sources of $C P$ violation. In addition, there is a great puzzle in cosmology known as the baryon symmetry of the Universe (BAU) [64]. CP violation has been discussed phenomenologically since the sixties. Gauge theories, naturally, account for its presence but leave its ultimate origin unanswered. Understanding the origin of $C P$ violation from first principles constitutes one of the central challenges of theoretical elementary particle physics. With the historic discovery of neutrino oscillations, the issue of leptonic $C P$ violation occupied the centre stage of the particle and nuclear physics. The existence of $C P$ violation in the lepton sector is expected in gauge theories of neutrino mass. The main difference with respect to $C P$ violation in the quark sector is the appearance of new phases associated with the Majorana nature of neutrinos and/or with the admixture of $S U(3) \times S U(2) \times U(1)$ singlet leptons in the charged-current weak interaction. The latter also leads to the effective violation of unitarity in the lepton mixing matrix describing neutrino oscillations. Irrespective of the underlying origin of neutrino mass, Majorana phases are a generic feature of gauge theories that account for the smallness of neutrino mass through the feebleness of lepton number violation.

Within the SM of particle physics, Yukawa couplings are free parameters which must span several orders of magnitude 
to accommodate hierarchical spectrum of fermion masses and mixings. The fermion masses and the mixings are derived from these Yukawa couplings. Also, radiative corrections can obscure the underlying structure. In the absence of complete knowledge of parameters appearing in the fermion mass matrices it is impossible, without some additional assumptions, to determine all the elements of the Yukawa coupling matrices for quarks and leptons. Therefore, without a significant breakthrough in the theoretical understanding of the fermion flavors, the phenomenological approaches are bound to play a crucial role in interpreting new experimental data on quark and lepton mixings. These approaches are expected to provide useful hints towards unraveling the dynamics of fermion mass generation, $C P$ violation, and identification of possible underlying symmetries of the lepton flavors from which realistic models of lepton mass generation and flavor mixing could, hopefully, be constructed. Even though the grand unification on its own does not shed any light on the flavor problem, the grand unified theories (GUTs) provide the optimal framework in which possible solutions to the flavor problem could be embedded. This is because the GUTs predict definite group theoretical relations between the fermion mass matrices. For this purpose, it is useful to find out possible leading order forms of the neutrino mass matrix. Such forms of neutrino mass matrix provide useful hints for model building which will eventually shed important light on the dynamics of lepton mass generation and flavor mixing. The first step in this direction is the reconstruction of the neutrino mass matrix in the flavor basis. However, the reconstruction results in a large variety of possible structures of mass matrices depending strongly on the mass scale, mass hierarchy, and the Majorana phases. There are several classes of models/Ansätze for Yukawa couplings. An incomplete list is as follows:

(1) approximate flavor symmetries [65-68] in which the entries in the Yukawa matrices are small parameters by which the flavor symmetries are broken;

(2) Fritzsch Ansätze and/or GUT inspired models [6974 ] in which some entries in the Yukawa mass matrices are assumed to be zero (e.g., by discrete flavor symmetry) and others may be related by some GUT relation;

(3) flavor democracy models $[75,76]$ in which all the entries in the Yukawa matrices are equal (i.e., no flavor symmetry) and hierarchy comes from diagonalization and renormalization group equation (RGE) running;

(4) string inspired models, composite models, and so forth.

In the context of type-I seesaw mechanism with three generations of right-handed neutrinos, which are singlets of $S U(2)$, after spontaneous symmetry breaking, the leptonic mass terms are given by

$$
\mathscr{L}=-\left[\overline{\nu_{L}^{0}} M_{D} \nu_{R}^{0}+v_{R}^{0 T} C M_{R} \nu_{R}^{0}+\overline{l_{L}^{0}} M_{l} l_{R}^{0}\right]+\text { h.c. }
$$

where $M_{R}, M_{D}$, and $M_{l}$ are right-handed Majorana neutrino, Dirac neutrino, and charged lepton mass matrices, respectively. The complex symmetric neutrino mass matrix, $M_{\gamma}$, is given by type I seesaw mechanism (equation (15)) [1316]. All the information about lepton masses and mixings is encoded in the hermitian charged lepton mass matrix $M_{l}$ and the complex symmetric neutrino mass matrix $M_{v}$.

In the basis where charged lepton mass matrix " $M_{l}$ " is diagonal, the neutrino mass matrix " $M_{\nu}$ " is diagonalized by a complex unitary matrix $V_{v}$

$$
M_{v}=V_{v} M_{v}^{\text {diag }} V_{v}^{T}
$$

where

$$
M_{v}^{\text {diag }}=\left(\begin{array}{ccc}
m_{1} & 0 & 0 \\
0 & m_{2} & 0 \\
0 & 0 & m_{3}
\end{array}\right)
$$

is the diagonal neutrino mass matrix. In the standard, Particle Data Group (PDG), parameterization of the neutrino mixing matrix, $V_{v}$, is given by

$$
\begin{aligned}
V_{v} \equiv U P & =\left(\begin{array}{ccc}
c_{12} c_{13} & s_{12} c_{13} & s_{13} e^{-i \delta} \\
-s_{12} c_{23}-c_{12} s_{13} s_{23} e^{i \delta} & c_{12} c_{23}-s_{12} s_{13} s_{23} e^{i \delta} & c_{13} s_{23} \\
s_{12} s_{23}-c_{12} s_{13} c_{23} e^{i \delta} & -c_{12} s_{23}-s_{12} s_{13} c_{23} e^{i \delta} & c_{13} c_{23}
\end{array}\right) \\
& \cdot\left(\begin{array}{ccc}
1 & 0 & 0 \\
0 & e^{i \alpha} & 0 \\
0 & 0 & e^{i(\beta+\delta)}
\end{array}\right),
\end{aligned}
$$

where $s_{i j}=\sin \theta_{i j}, c_{i j}=\cos \theta_{i j}$, and $P$ is the Majorana phase matrix. In addition to the Majorana phases $\alpha, \beta$, the phase matrix contains Dirac-type phase $\delta$ just to make $(1,1)$ element of $M_{v}\left(m_{e e}\right)$ independent of $\delta$. The neutrino mass matrix " $M_{\nu}$ " can be written as

$$
M_{\nu}=U P M_{\nu}^{\text {diag }} P^{T} U^{T} .
$$

In terms of the neutrino masses, mixing angles and $C P$ violating phases, the neutrino mass matrix " $M_{\nu}$ " is given by

$$
M_{\nu}=\left(\begin{array}{ccc}
m_{e e} & m_{e \mu} & m_{e \tau} \\
m_{e \mu} & m_{\mu \mu} & m_{\mu \tau} \\
m_{e \tau} & m_{\mu \tau} & m_{\tau \tau}
\end{array}\right),
$$

where

$$
\begin{gathered}
m_{e e}=c_{12}^{2} c_{13}^{2} m_{1}+c_{13}^{2} e^{2 i \alpha} m_{2} s_{12}^{2}+e^{2 i \beta} m_{3} s_{13}^{2} \\
m_{e \mu}=c_{13}\left(e^{i(2 \beta+\delta)} m_{3} s_{13} s_{23}-c_{12} m_{1}\left(c_{23} s_{12}+c_{12} e^{i \delta} s_{13} s_{23}\right)\right. \\
\left.+e^{2 i \alpha} m_{2} s_{12}\left(c_{12} c_{23}-e^{i \delta} s_{12} s_{13} s_{23}\right)\right), \\
m_{e \tau}=c_{13}\left(c_{23} e^{i(2 \beta+\delta)} m_{3} s_{13}-e^{2 i \alpha} m_{2} s_{12}\left(c_{23} e^{i \delta} s_{12} s_{13}+c_{12} s_{23}\right)\right. \\
\left.+c_{12} m_{1}\left(-c_{12} c_{23} e^{i \delta} s_{13}+s_{12} s_{23}\right)\right),
\end{gathered}
$$




$$
\begin{aligned}
m_{\mu \mu}= & c_{13}^{2} e^{2 i(\beta+\delta)} m_{3} s_{23}^{2}+m_{1}\left(c_{23} s_{12}+c_{12} e^{i \delta} s_{13} s_{23}\right)^{2} \\
& +e^{2 i \alpha} m_{2}\left(c_{12} c_{23}-e^{i \delta} s_{12} s_{13} s_{23}\right)^{2} \\
m_{\mu \tau}= & c_{13}^{2} c_{23} e^{2 i(\beta+\delta)} m_{3} s_{23}+m_{1}\left(c_{12} c_{23} e^{i \delta} s_{13}-s_{12} s_{23}\right) \\
& \cdot\left(c_{23} s_{12}+c_{12} e^{i \delta} s_{13} s_{23}\right) \\
& -e^{2 i \alpha} m_{2}\left(c_{23} e^{i \delta} s_{12} s_{13}+c_{12} s_{23}\right) \\
& \cdot\left(c_{12} c_{23}-e^{i \delta} s_{12} s_{13} s_{23}\right) \\
m_{\tau \tau}= & c_{13}^{2} c_{23}^{2} e^{2 i(\beta+\delta)} m_{3}+e^{2 i \alpha} m_{2}\left(c_{23} e^{i \delta} s_{12} s_{13}+c_{12} s_{23}\right)^{2} \\
+ & m_{1}\left(c_{12} c_{23} e^{i \delta} s_{13}-s_{12} s_{23}\right)^{2} .
\end{aligned}
$$

It can be seen from (53) and (54) that the neutrino mass matrix contains nine physical parameters, namely, the three neutrino mass eigenvalues $\left(m_{1}, m_{2}\right.$, and $\left.m_{3}\right)$, three mixing angles $\left(\theta_{12}, \theta_{23}\right.$, and $\left.\theta_{13}\right)$, and three $C P$ violating phases $\delta, \alpha$, and $\beta$. Out of these nine free parameters of the theory not all are measured experimentally (Table 3 ). In particular, the $C P$ violating phases are completely unconstrained. Thus, it is impossible to fully reconstruct the neutrino mass matrix from the results of feasible experiments and, thus, one cannot be definite about the origin and magnitude of $C P$ violation in the leptonic sector. However, as a first step, it is worthwhile to investigate the neutrino mass matrix in the presence of some theoretically motivated inputs imposed on $M_{v}$ so as to reduce the number of free parameters of the theory and induce relationships amongst them. The possible forms of these theoretically motivated inputs are constrained by the current experimental data on neutrino masses and mixings and include the imposition of "texture zeros" [77-83], "hybrid textures" [84-86] in the neutrino mass matrix, requirement of zero determinant $[87,88]$, and zero trace condition [89] to name just a few.

In the texture zero Ansätze, it is assumed that some of the elements of neutrino mass matrix are anomalously small as compared to the other elements and can be, effectively, replaced by zeros. This is the simplest procedure to reduce the number of free parameters in the mass matrix. The texture Ansätze has been quite successful in the quark sector. A phenomenologically favored texture of quark mass matrix has been presented earlier by Gupta and Rajpoot [90-93]. In the spirit of quark-lepton similarity, the same texture has been prescribed for the charged lepton and the Dirac neutrino mass matrices [94]. The same texture for the righthanded neutrino mass matrix in the seesaw mechanism might follow from universal flavor symmetry hidden in a more fundamental theory of mass generation. Thus, texture zeros in different positions of the neutrino mass matrix, in particular and fermion mass matrices, in general could be the consequence of an underlying symmetry $[95,96]$. Such universal textures of fermion mass matrices can, theoretically, be obtained in the context of GUTs based on $S O(10)$ [97].
Moreover, neutrino mass matrices with texture zeros have important implications for leptogenesis [98].

Some attempts aimed at understanding the pattern of quark/lepton masses and mixings by introducing Abelian or non-Abelian flavor symmetries, naturally, lead to texture zeros in the mass matrices. However, the current low energy data are consistent only with a limited number of texture zero schemes of the neutrino mass matrix. They can, also, be realized within the framework of the seesaw mechanism. Furthermore, these textures can also be derived from a simple flavor group $A_{4}$ or its $Z_{3}$ subgroup [99]. The current neutrino oscillation data disallow all neutrino mass matrices with three or more texture zeros in the flavor basis. Out of fifteen possible neutrino mass matrices with two texture zeros, only seven are compatible with the current data on neutrino masses and mixings. Some sets of these texture zeros can be obtained by suitable weak basis (WB) transformations and have no physical meaning as such. However, a large class of sets of leptonic texture zeros considered in the literature imply the vanishing of certain $C P$-odd WB invariants and one can, thus, recognize a lepton flavor model in which the texture zeros are not explicitly present but correspond to a particular texture structure in a certain WB. The presence of texture zeros, in general, leads to a decrease in the number of independent $C P$ violating phases. A particular texture zero structure gives rise to definite relationships between different $C P$ violating phases $[100,101]$. Such exact relations in closed form were obtained in [79]. Correlations between Dirac- and Majorana-type $C P$ violating phases for a particular texture zero scheme were studied in detail in $[100,101]$. It is, therefore, important to examine the interrelationships between the $C P$ odd WB invariants which are required to vanish as a necessary and sufficient condition for $C P$ conservation.

Moreover, a unified description of flavor physics and $C P$ violation in the quark and lepton sectors is absolutely necessary. This can be achieved by constructing a low energy effective theory with the SM and some discrete non-Abelian family symmetry and, subsequently, embedding this theory into grand unified theory (GUT) models like $S O(10)$ [97]. For this reason, the discrete symmetry will have to be a subgroup of $S O(3)$ or $S U(3)$ [102]. The search for an adequate discrete symmetry has mainly focussed on the minimal subgroups of these groups with at least one singlet and one doublet irreducible representation to accommodate the fermions belonging to each generation. One such subgroup is the quaternion group $Q_{8}$ [85] which not only accommodates the three generations of fermions but also explains the rather large difference between the values of 2-3 mixings in the quark and lepton sectors. Quaternion symmetry like some other discrete symmetries leads to nontrivial relationships amongst the nonzero mass matrix elements which underscores the inadequacy of texture zero analyses [84$86]$ in isolation. Such textures which have equalities between different elements along with the vanishing of some elements of the mass matrix have been referred to as "hybrid textures" in the literature. Frigerio and Smirnov [86] presented a comprehensive analysis of the hybrid textures along with other possibilities for the neutrino mass matrix. The discrete quaternion groups have been extensively applied to flavor 
physics. It is, therefore, extremely important to investigate the connection between the textures of fermion mass matrices and the observables of flavor mixing. In particular, it is important to subject models based on $Q_{8}$ symmetry to the test of a viable leptogenesis to explain the baryon asymmetry of the Universe (BAU).

The phenomenological analyses of the neutrino mass matrix with "texture zeros" or "hybrid textures" are expected to provide useful hints towards unraveling the dynamics of fermion mass generation, $C P$ violation, and identification of possible underlying symmetries of the lepton flavors from which realistic models of lepton mass generation and flavor mixing could be, hopefully, constructed. Moreover, an interesting cosmological implication of leptonic $C P$ violation is that it opens an attractive possibility of accounting for the matter-antimatter asymmetry in the Universe within the socalled thermal leptogenesis mechanism.

\section{Sterile Neutrinos and Associated Phenomenology}

The recent agreement of MiniBooNE antineutrino data [103] with the short-baseline $\bar{\nu}_{\mu} \rightarrow \bar{\nu}_{e}$ oscillation signal observed by LSND experiment [104] has opened an intense theoretical and experimental activity aimed at the clarification of the explanation of these observations in a framework compatible with the data of other neutrino oscillation experiments. The results of solar, atmospheric, and long-baseline neutrino oscillation experiments led us to the current standard threeneutrino mixing paradigm, in which the three active neutrinos $v_{e}, v_{\mu}, v_{\tau}$ are superpositions of three massive neutrinos $v_{1}, v_{2}, v_{3}$ with masses $m_{1}, m_{2}, m_{3}$, respectively. The measured solar and atmospheric mass-squared dofferences are given in Table 3. The completeness of this paradigm has been challenged by the LSND [104] and MiniBooNE observations of short-baseline $\bar{\nu}_{\mu} \rightarrow \bar{\nu}_{e}$ transitions at different values of distance $(L)$ and energy $(E)$ but approximately at the same $L / E$. Since the distance and energy dependences of neutrino oscillations occur through this ratio, the agreement of MiniBooNE and LSND signals raised interest in the possibility of existence of one or more mass squared differences larger than about $0.5 \mathrm{eV}$, which is much larger than the values of $\Delta m_{21}^{2}$ and $\Delta m_{31}^{2}$. Hence, we are led to the extension of threeneutrino mixing with the introduction of one or more sterile neutrinos which do not have weak interactions and do not contribute to the invisible width of the $Z$ boson [105].

The possible existence of sterile neutrinos is an exciting possibility which could open a powerful window to investigate physics beyond the SM of particle physics. Schemes of neutrino mixing with sterile neutrinos have been studied extensively in the literature ([106] and the references therein) with more emphasis on the schemes with one or two sterile neutrinos. Sterile neutrinos, if they exist, would lead to rich experimental phenomena. Their admixture with active neutrinos would modify the neutrino flavor mixing and lead to the observable effects in neutrino oscillation experiments. Moreover, they could interact with gauge bosons, resulting in significant corrections to certain nonoscillation processes, for example, in the neutrinoless double beta decay $(0 \nu \beta \beta)$ amplitude [107] or in the beta decay spectra such as in KATRIN experiment $[108,109]$.

In the presence of $n_{s}=n-3$ sterile neutrinos, the neutrino mass matrix is an $n \times n$ matrix $M_{v}$ which can be diagonalized by an $n \times n$ unitary matrix $U$. The neutrino flavor eigenstates $\nu_{f}$ (for $f=e, \mu, \tau, s_{1}, s_{2}, \ldots, s_{n-3}$ ) are then related to their mass eigenstates $v_{i}\left(\nu_{1}, v_{2}, v_{3}, v_{s_{1}}, v_{s_{2}}, \ldots\right)$ (for $i=1,2,3, \ldots, n$ ) via

$$
\nu_{f}=\sum_{i=1}^{n} U_{f i} \nu_{i} .
$$

In general, for $n$ massive families including $n_{s}=n-3 \neq 0$ massive sterile neutrinos, one has $n-1=n_{s}+2$ Majorana phases, $3(n-2)=3\left(n_{s}+1\right)$ mixing angles, and $2 n-5=2 n_{s}+1$ Dirac phases. The number of angles and Dirac phases is less than the naive $(1 / 2) n(n-1)$ angles and $(1 / 2)(n-1)(n-2)$ phases because the $(1 / 2) n_{s}\left(n_{s}-1\right)$ rotations between sterile states are unphysical. For illustration, in the case of only one sterile neutrino, $U$ is typically parameterized by

$$
U=R_{34} \widetilde{R_{24}} \widetilde{R_{14}} R_{23} \widetilde{R_{13}} R_{12} P,
$$

where the matrices $R_{i j}$ are rotations in $i j$ space; that is,

$$
R_{34}=\left(\begin{array}{cccc}
1 & 0 & 0 & 0 \\
0 & 1 & 0 & 0 \\
0 & 0 & c_{34} & s_{34} \\
0 & 0 & -s_{34} & c_{34}
\end{array}\right)
$$

or

$$
\widetilde{R_{14}}=\left(\begin{array}{cccc}
c_{14} & 0 & 0 & s_{14} e^{-i \delta_{14}} \\
0 & 1 & 0 & 0 \\
0 & 0 & 1 & 0 \\
-s_{14} e^{i \delta_{14}} & 0 & 0 & c_{14}
\end{array}\right)
$$

where $s_{i j}=\sin \theta_{i j}, c_{i j}=\cos \theta_{i j}$. The diagonal $P$ matrix contains the three Majorana phases $\alpha, \beta$, and $\gamma$ :

$$
P=\operatorname{diag}\left(1, e^{i \alpha / 2}, e^{i\left(\beta / 2+\delta_{13}\right)}, e^{i\left(\gamma / 2+\delta_{14}\right)}\right) .
$$

Note that there are in total three Dirac $C P$-violating phases $\delta_{i j}$. The above definition of $P$ is constructed in such a way that only Majorana phases show up in the effective mass governing neutrino-less double beta decay. Similarly, one can parameterize the mixing matrix for 2 sterile neutrinos as

$$
U=\widetilde{R_{25}} R_{34} R_{25} \widetilde{R_{24}} R_{23} \widetilde{R_{15}} \widetilde{R_{14}} \widetilde{R_{13}} R_{12} P
$$

where

$$
P=\operatorname{diag}\left(1, e^{i \alpha / 2}, e^{i\left(\beta / 2+\delta_{13}\right)}, e^{i\left(\gamma / 2+\delta_{14}\right)} e^{i\left(\phi / 2+\delta_{15}\right)}\right) .
$$

Neutrino-less double beta decay $(0 \nu \beta \beta)$ is the only realistic test of lepton number violation. While there are several mechanisms to mediate the process (e.g., heavy neutrinos, 
right-handed currents, or SUSY particles), light Majorana neutrino exchange is presumably the best motivated scenario. In the presence of one and two sterile neutrinos, the effective neutrino mass in $(0 \nu \beta \beta)$ is, respectively, given by

$$
\begin{aligned}
\left\langle m_{e e}\right\rangle_{1}= & \mid c_{12}^{2} c_{13}^{2} c_{14}^{2} m_{1}+s_{12}^{2} c_{13}^{2} c_{14}^{2} m_{2} e^{i \alpha} \\
& +s_{13}^{2} c_{14}^{2} m_{3} e^{i \beta}+s_{14}^{2} m_{4} e^{i \gamma} \mid, \\
\left\langle m_{e e}\right\rangle_{2}=\mid & c_{12}^{2} c_{13}^{2} c_{14}^{2} c_{15}^{2} m_{1}+s_{12}^{2} c_{13}^{2} c_{14}^{2} c_{15}^{2} m_{2} e^{i \alpha} \\
& +s_{13}^{2} c_{14}^{2} c_{15}^{2} m_{3} e^{i \beta}+s_{14}^{2} c_{15}^{2} m_{4} e^{i \gamma}+s_{15}^{2} m_{5} e^{i \phi} \mid .
\end{aligned}
$$

\section{Conclusions}

In the absence of significant breakthrough in our understanding of the parameters of neutrino mass matrix, the phenomenological approaches such as texture zeros, hybrid textures, zero determinant, and zero trace condition, to name a few, are bound to play important role in our understanding of neutrino masses and mixings. This in turn is intimately related to the possible observation $C P$ violation in the leptonic sector. In this direction, recent observation of nonzero $\theta_{13}$ has very important implication for $C P$ violation in the leptonic sector as it requires a nonzero value of $\theta_{13}$. The texture zeros are not weak basis (WB) invariants. This means that a given set of texture zeros which arise in a certain WB may not be present at all or may appear in different entries in another WB. A large class of sets of leptonic texture zeros considered in the literature imply the vanishing of certain $C P$-odd weak basis invariants. Thus, we can recognize a lepton mass model in which the texture zeros are not explicitly present and which corresponds to a particular texture scheme in a certain WB. The relevance of $C P$-odd WB invariants in the analysis of the texture zero Ansätze is due to the fact that texture zeros lead to a decrease in the number of the independent $C P$ violating phases. A minimum number of $C P$-odd WB invariants can be found which will all vanish for the $C P$ invariant mass matrices as a necessary and sufficient condition. Despite the success of SM threeneutrino oscillations in explaining the results of solar, atmospheric, reactor, and accelerator experiments by two large and one small mixing angles and two distinct mass squared splittings, MiniBooNE and LSND experiments are hinting to the existence of extra mass squared splittings and therefore the mixing of the three SM active neutrinos with extra sterile neutrinos. The existence of sterile neutrinos greatly influencess the phenomenology of the neutrino mass matrix as it would change the neutrino oscillation probabilities. Furthermore, there would exist new sources of $C P$ violation affecting the amplitude of neutrinoless double beta decay $(0 \nu \beta \beta)$. In view of the recent enthusiastic results obtained from the experiments and the future experiments which are at the planning stage, the future of the neutrino physics is very buoyant and the next step is the observation of $C P$ violation in the leptonic sector which is very important for the particle and astroparticle points of view.

\section{Conflict of Interests}

The author declares that there is no conflict of interests regarding the publication of this paper.

\section{Acknowledgments}

The author acknowledges the financial support provided by University Grants Commission (UGC)-Basic Science Research (BSR), Government of India vide Grant no. F.202(30)/2013(BSR).

\section{References}

[1] L. Meitner and O. Hahn, "Über die Verteilung der BetaStrahlen auf die einzelnen Produkte des aktiven Niederschlags des Thoriums," Physikalische Zeitschrift, vol. 13, p. 390, 1912.

[2] J. Chadwick, "Distribution in intensity in the magnetic spectrum of the $\beta$-rays of radium," Verhandlungen der Deutschen Physikalischen Gesellschaft, vol. 16, pp. 383-391, 1914.

[3] W. Pauli, "Dear radioactive ladies and gentlemen," in Proceedings of the Tubingen conference, December 1930.

[4] S. F. King, A. Merle, S. Morisi, Y. Shimizu, and M. Tanimoto, "Neutrino mass and mixing: from theory to experiment," New Journal of Physics, vol. 16, Article ID 045018, 2014.

[5] S. M. Bilenky, "Neutrino in standard model and beyond," http://arxiv.org/abs/1501.00232.

[6] S. M. Bilenky and B. Pontecorvo, "Lepton mixing and neutrino oscillations," Physics Reports, vol. 41, no. 4, pp. 225-261, 1978.

[7] S. M. Bilenky and S. T. Petcov, "Massive neutrinos and neutrino oscillations," Reviews of Modern Physics, vol. 59, no. 3, pp. 671754, 1987.

[8] S. M. Bilenky, C. Giunti, and W. Grimus, "Phenomenology of neutrino oscillations," Progress in Particle and Nuclear Physics, vol. 43, no. 1, pp. 1-86, 1999.

[9] S. M. Bilenky, C. Giunti, J. A. Grifols, and E. Massó, "Absolute values of neutrino masses: status and prospects," Physics Reports, vol. 379, no. 2, pp. 69-148, 2003.

[10] M. Gonzalez-Garcia and Y. Nir, "Neutrino masses and mixing: evidence and implications," Reviews of Modern Physics, vol. 75, no. 2, pp. 345-402, 2003.

[11] M. Maltoni, T. Schwetz, M. Tórtola, and J. W. F. Valle, "Status of global fits to neutrino oscillations," New Journal of Physics, vol. 6, p. 122, 2004.

[12] G. L. Fogli, E. Lisi, A. Marrone, and A. Palazzo, "Global analysis of three-flavor neutrino masses and mixings," Progress in Particle and Nuclear Physics, vol. 57, no. 2, pp. 742-795, 2006.

[13] T. Yanagida, in Proceedings of the Workshop on the Baryon Number of the Universe and Unified Theories, Tsukuba, Japan, February 1979.

[14] P. Minkowski, " $\mu \rightarrow e \gamma$ at a rate of one out of 109 muon decays?” Physics Letters B, vol. 67, no. 4, pp. 421-428, 1977.

[15] M. Gell-Mann, P. Ramond, and R. Slansky, Supergravity, NorthHolland, Amsterdam, The Netherlands, 1979.

[16] R. N. Mohapatra and G. Senjanović, "Neutrino mass and spontaneous parity nonconservation," Physical Review Letters, vol. 44, no. 14, pp. 912-915, 1980.

[17] M. Kobayashi and T. Maskawa, "CP-violation in the renormalizable theory of weak interaction," Progress of Theoretical Physics, vol. 49 , no. 2, pp. 652-657, 1973. 
[18] R. Davis, D. S. Harmer, and K. C. Hoffman, "Search for neutrinos from the sun," Physical Review Letters, vol. 20, no. 21, pp. 1205-1209, 1968.

[19] S. P. Mikheyev and A. Y. Smirnov, "Resonant amplification of $v$ oscillations in matter and solar-neutrino spectroscopy," Il Nuovo Cimento C, vol. 9, no. 1, pp. 17-26, 1986.

[20] L. Wolfenstein, "Neutrino oscillations in matter," Physical Review D, vol. 17, no. 9, pp. 2369-2374, 1978.

[21] V. Gribov and B. Pontecorvo, "Neutrino astronomy and lepton charge," Physics Letters B, vol. 28, no. 7, pp. 493-496, 1969.

[22] Y. Fukuda, T. Hayakawaa, E. Ichiharaa et al., "Evidence for oscillation of atmospheric neutrinos," Physical Review Letters, vol. 81, no. 8, pp. 1562-1567, 1998.

[23] Y. Fukuda, T. Hayakawa, E. Ichihara et al., "Study of the atmospheric neutrino ux in the multi-GeV energy range," Physics Letters B, vol. 436, pp. 33-41, 1998.

[24] Q. R. Ahmad, R. C. Allen, T. C. Andersen et al., "Measurement of the rate of $v_{e}+d \rightarrow p+p+e^{-}$interactions produced by ${ }^{8} B$ solar neutrinos at the sudbury neutrino observatory," Physical Review Letters, vol. 87, no. 7, Article ID 071301, 6 pages, 2001.

[25] Q. R. Ahmad, R. C. Allen, T. C. Andersen et al., "Direct evidence for neutrino flavor transformation from neutral-current interactions in the sudbury neutrino observatory," Physical Review Letters, vol. 89, Article ID 011301, 2002.

[26] K. Eguchi, S. Enomoto, K. Furuno et al., "First results from KamLAND: evidence for reactor antineutrino disappearance," Physical Review Letters, vol. 90, Article ID 021802, 2003.

[27] S. J. Parke, "Nonadiabatic level crossing in resonant neutrino oscillations," Physical Review Letters, vol. 57, no. 10, pp. 12751278, 1986.

[28] B. T. Cleveland, T. Daily, R. Davis Jr. et al., "Measurement of the solar electron neutrino flux with the homestake chlorine detector," The Astrophysical Journal, vol. 496, p. 505, 1998.

[29] J. N. Abdurashitov, E. P. Veretenkin, V. M. Vermul et al., "Solar neutrino flux measurements by the Soviet-American gallium experiment (SAGE) for half the 22-year solar cycle," Journal of Experimental and Theoretical Physics, vol. 95, no. 2, pp. 181-193, 2002.

[30] J. N. Abdurashitov, "Solar neutrino flux measurements by the Soviet-American gallium experiment (SAGE) for half the 22year solar cycle," Zhurnal Eksperimental'noi i Teoreticheskoi Fiziki, vol. 122, pp. 211-226, 2002.

[31] W. Hampel, J. Handt, G. Heusser et al., "GALLEX solar neutrino observations: results for GALLEX IV," Physics Letters B, vol. 447, no. 1-2, pp. 127-133, 1999.

[32] S. Fukuda, Y. Fukudaa, M. Ishitsuka et al., "Determination of solar neutrino oscillation parameters using 1496 days of SuperKamiokande-I data," Physics Letters B, vol. 539, no. 3-4, pp. 179$187,2002$.

[33] C. Arpesella, G. Bellini, J. Benziger et al., "First real time detection of ${ }^{7} \mathrm{Be}$ solar neutrinos by Borexino," Physics Letters B, vol. 658, no. 4, pp. 101-108, 2008.

[34] T. Araki, K. Eguchi, S. Enomoto et al., "Measurement of neutrino oscillation with KamLAND: evidence of spectral distortion," Physical Review Letters, vol. 94, no. 8, Article ID 081801, 5 pages, 2005.

[35] S. Abe, T. Ebihara, S. Enomoto et al., "Precision measurement of neutrino oscillation parameters with KamLAND," Physical Review Letters, vol. 100, no. 22, Article ID 221803, 5 pages, 2008.

[36] I. Shimizu, in Proceedings of the International Conference on Topics in Astroparticle and Underground Physics (TAUP'07), 2007.
[37] M. C. Gonzalez-Garcia and M. Maltoni, "Phenomenology with massive neutrinos," Physics Reports, vol. 460, no. 1-3, pp. 1-129, 2008.

[38] M. Maltoni, T. Schwetz, M. A. Tortola, and J. W. F. Valle, "Status of global fits to neutrino oscillations," New Journal of Physics, vol. 6, no. 1, article 122, 2004.

[39] S. Goswami, "Neutrino oscillations and masses," International Journal of Modern Physics A, vol. 21, pp. 1901-1913, 2006.

[40] A. Bandyopadhyay, S. Choubey, S. Goswami, S. T. Petcov, and D. P. Roy, "Update of the solar neutrino oscillation analysis with the 766 Ty KamLAND spectrum," Physics Letters B, vol. 608, no. 1-2, pp. 115-129, 2005.

[41] G. L. Fogli, E. Lisi, A. Marrone et al., "Observables sensitive to absolute neutrino masses: a reappraisal after WMAP 3-year and first MINOS results," Physical Review D, vol. 75, Article ID 053001, 2007.

[42] Y. Ashie, J. Hosaka, K. Ishihara et al., "Measurement of atmospheric neutrino oscillation parameters by Super-Kamiokande I," Physical Review D, vol. 71, Article ID 112005, 2005.

[43] M. H. Ahn, S. Aoki, H. Bhang et al., "Indications of neutrino oscillation in a $250 \mathrm{~km}$ long-baseline experiment," Physical Review Letters, vol. 90, no. 4, Article ID 041801, 5 pages, 2003.

[44] E. Aliu, S. Andringa, S. Aoki et al., "Evidence for muon neutrino oscillation in an accelerator-based experiment," Physical Review Letters, vol. 94, no. 8, Article ID 081802, p. 5, 2005.

[45] D. G. Michael, P. Adamson, T. Alexopoulos et al., "Observation of muon neutrino disappearance with the MINOS detectors in the NuMI neutrino beam," Physical Review Letters, vol. 97, Article ID 191801, 2006.

[46] J. I. Crespo-Anadon, "Double Chooz: latest results," http://arxiv. org/abs/1412.3698.

[47] Y. Abe, C. Aberle, J. C. dos Anjos et al., "Reactor $\bar{\nu}_{\mathrm{e}}$ disappearance in the double chooz experiment," Physical Review D, vol. 86, no. 5, Article ID 052008, 21 pages, 2012.

[48] Y. Abe, C. Aberle, J. C. dos Anjos et al., "First measurement of $\theta 13$ from delayed neutron capture on hydrogen in the Double Chooz experiment," Physics Letters B, vol. 723, no. 1-3, pp. 6670, 2013.

[49] Y. Abe, J. C. dos Anjose, J. C. Barriere et al., "Backgroundindependent measurement of $\boldsymbol{\theta}_{13}$ in Double Chooz," Physical Letters B, vol. 735, pp. 51-56, 2014.

[50] Y. Abe, J. C. dos Anjos, J. C. Barriere et al., "Ortho-positronium observation in the Double Chooz Experiment," Journal of High Energy Physics, vol. 2014, article 32, 2014.

[51] F. P. An, A. B. Balantekin, H. R. Band et al., "Spectral measurement of electron antineutrino oscillation amplitude and frequency at Daya Bay," Physical Review Letters, vol. 112, Article ID 061801, 2014.

[52] P. Adamson, I. Anghel, C. Backhouse et al., "Electron neutrino and antineutrino appearance in the full MINOS data sample," Physical Review Letters, vol. 110, no. 17, Article ID 171801, 2013.

[53] K. Abe, J. Adam, H. Aihara et al., "Observation of electron neutrino appearance in a muon neutrino beam," Physical Review Letters, vol. 112, Article ID 061802, 2014.

[54] M. C. Gonzalez-Garcia, M. Maltoni, and T. Schwetz, "Updated fit to three neutrino mixing: status of leptonic CP violation," Journal of High Energy Physics, vol. 2014, article 52, 2014.

[55] N. Cabibbo, "Unitary symmetry and leptonic decays," Physical Review Letters, vol. 10, pp. 531-533, 1963.

[56] A. H. Guth and P. J. Steinhardt, "The inflationary universe," Scientific American, vol. 250, no. 5, pp. 90-102, 1984. 
[57] L. Fromme, S. J. Huber, and M. Seniuch, "Baryogenesis in the two-Higgs doublet model," Journal of High Energy Physics, vol. 2006, no. 11, p. 038, 2006.

[58] K. Kajantie, M. Laine, K. Rummukainen, and M. E. Shaposhnikov, "The electroweak phase transition: a non-perturbative analysis," Nuclear Physics B, vol. 466, no. 1-2, pp. 189-258, 1996.

[59] C. Jarlskog, "Commutator of the quark mass matrices in the standard electroweak model and a measure of maximal CP nonconservation," Physical Review Letters, vol. 55, no. 10, pp. 1039-1042, 1985.

[60] C. Amsler, M. Doser, M. Antonelli et al., "Review of particle physics," Physics Letters B, vol. 667, no. 1-5, pp. 1-6, 2008.

[61] G. C. Branco, L. Lavoura, and M. N. Rebelo, "Majorana neutrinos and CP violation in the leptonic sector," Physics Letters B, vol. 180 , no. 3, pp. 264-268, 1986.

[62] G. C. Branco and L. Lavoura, "On the addition of vector-like quarks to the standard model," Nuclear Physics B, vol. 278, pp. 738-754, 1986.

[63] H. K. Dreiner, J. S. Kim, O. Lebedev, and M. Thormeier, "Supersymmetric Jarlskog invariants: the neutrino sector," Physical Review D: Particles, Fields, Gravitation and Cosmology, vol. 76, no. 1, Article ID 015006, 2007.

[64] A. D. Sakharov, "Violation of CP invariance, C asymmetry, and baryon asymmetry of the universe," ZhETF Pis ma Redaktsiiu, vol. 5, p. 32, 1967.

[65] C. D. Froggat and H. B. Nielsen, "Hierarchy of quark masses, cabibbo angles and CP violation," Nuclear Physics B, vol. 147, no. 3-4, pp. 277-298, 1979.

[66] C. D. Froggatt and H. B. Nielsen, "Statistical analysis of quark and lepton masses," Nuclear Physics B, vol. 164, pp. 114-140, 1980.

[67] A. Antaramian, L. J. Hall, and A. Rašin, "Flavor-changing interactions mediated by scalars at the weak scale," Physical Review Letters, vol. 69, no. 13, pp. 1871-1873, 1992.

[68] S. F. King and C. Luhn, "Neutrino mass and mixing with discrete symmetry," Reports on Progress in Physics, vol. 76, no. 5, Article ID 056201, 2013.

[69] H. Fritzsch, "Weak-interaction mixing in the six-quark theory," Physics Letters B, vol. 73, no. 3, pp. 317-322, 1978.

[70] H. Fritzsch, "Quark masses and flavor mixing," Nuclear Physics $B$, vol. 155, pp. 189-207, 1979.

[71] S. Dimopoulos, "Natural generation of fermion masses," Physics Letters B, vol. 129, no. 6, pp. 417-428, 1983.

[72] T. P. Chang and M. Sher, "Mass-matrix ansatz and flavor nonconservation in models with multiple Higgs doublets," Physical Review D, vol. 35, no. 11, pp. 3484-3491, 1987.

[73] H. Georgi and C. Jarlskog, "A new lepton-quark mass relation in a unified theory," Physics Letters B, vol. 86, no. 3-4, pp. 297-300, 1979.

[74] S. Dimopoulos, L. J. Hall, and S. Raby, "Predictive framework for fermion masses in supersymmetric theories," Physical Review Letters, vol. 68, no. 13, pp. 1984-1987, 1992.

[75] H. Harari, H. Haut, and J. Weyers, "Quark masses and cabibbo angles," Physics Letters B, vol. 78, no. 4, pp. 459-461, 1978.

[76] H. Fritzsch and J. Plankl, "Flavour democracy and the leptonquark hierarchy," Physics Letters, Section B: Nuclear, Elementary Particle and High-Energy Physics, vol. 237, no. 3-4, pp. 451-456, 1990.

[77] P. H. Frampton, S. L. Glashow, and D. Marfatia, "Zeroes of the neutrino mass matrix," Physics Letters, Section B: Nuclear, Elementary Particle and High-Energy Physics, vol. 536, no. 1-2, pp. 79-82, 2002.
[78] B. R. Desai, D. P. Roy, and A. R. Vaucher, "Three-neutrino mass matrices with two texture zeros," Modern Physics Letters A, vol. 18, no. 20, pp. 1355-1365, 2003.

[79] Z.-Z. Xing, "Texture zeros and majorana phases of the neutrino mass matrix," Physics Letters B, vol. 530, no. 1-4, pp. 159-166, 2002.

[80] W. Guo and Z.-Z. Xing, "Implications of the KamLAND measurement on the lepton flavor mixing matrix and the neutrino mass matrix," Physical Review D, vol. 67, Article ID 053002, 2003.

[81] A. Merle and W. Rodejohann, "Elements of the neutrino mass matrix: allowed ranges and implications of texture zeros," Physical Review D, vol. 73, no. 7, Article ID 073012, 11 pages, 2006.

[82] S. Dev and S. Kumar, "Neutrino parameter space for a vanishing ee element in the neutrino mass matrix," Modern Physics Letters A, vol. 22, p. 1401, 2007.

[83] M. Honda, S. Kaneko, and M. Tanimoto, "Prediction of U e3 in neutrino mass matrix with two zeros," Journal of High Energy Physics, vol. 2003, no. 9, article 28, 2003.

[84] S. Kaneko, H. Sawanaka, and M. Tanimoto, "Hybrid textures of neutrinos," Journal of High Energy Physics, vol. 2005, no. 8, article 73, 2005.

[85] M. Frigerio, S. Kaneko, E. Ma, and M. Tanimoto, "Quaternion family symmetry of quarks and leptons," Physical Review D, vol. 71, Article ID 011901, 2005.

[86] M. Frigerio and A. Y. Smirnov, "Neutrino mass matrix: inverted hierarchy and CP violation," Physical Review D, vol. 67, Article ID $013007,2003$.

[87] G. C. Branco, R. González Felipe, F. R. Joaquim, and T. Yanagida, "Removing ambiguities in the neutrino mass matrix," Physics Letters B, vol. 562, no. 3-4, pp. 265-272, 2003.

[88] B. C. Chauhan, J. Pulido, and M. Picariello, "Neutrino mass matrices with vanishing determinant," Physical Review DParticles, Fields, Gravitation and Cosmology, vol. 73, no. 5, Article ID 053003, 2006.

[89] X.-G. He and A. Zee, "Neutrino masses with a 'zero sum' condition: $m_{v_{1}}+m_{v_{2}}+m_{v_{3}}=0$," Physical Review D, vol. 68, no. 3, Article ID 037302, p. 4, 2003.

[90] S. N. Gupta and S. Rajpoot, Quark Mass Matrices and the Top Quark Model, Wayne State University, September 1990.

[91] S. N. Gupta and J. M. Johnson, "Quark mixing and CP violation," Physical Review D, vol. 44, no. 7, pp. 2110-2113, 1991.

[92] S. Rajpoot, "Evading the top mass bound in the fritzsch scheme," Modern Physics Letters A, vol. 7, no. 4, pp. 309-313, 1992.

[93] H. Fritzsch and Z.-Z. Xing, "A symmetry pattern of maximal $C P$ violation and a determination of the unitarity triangle," Physics Letters B, vol. 353, pp. 114-118, 1995.

[94] G. Ahuja, S. Kumar, M. Randhawa, M. Gupta, and S. Dev, "Texture 4 zero Fritzsch-like lepton mass matrices," Physical Review D: Particles, Fields, Gravitation and Cosmology, vol. 76, no. 1, Article ID 013006, 2007.

[95] P. H. Frampton, M. C. Oh, and T. Yoshikawa, "Majorana mass zeros from Higgs triplet vacuum expectation values without a Majoron problem," Physical Review D, vol. 66, Article ID 033007, 2002.

[96] W. Grimus and L. Lavoura, "On a model with two zeros in the neutrino mass matrix," Journal of Physics G: Nuclear and Particle Physics, vol. 31, no. 7, pp. 693-702, 2005.

[97] H. S. Goh, R. N. Mohapatra, and S.-P. Ng, "Minimal supersymmetric $\mathrm{SO}(10)$ model and predictions for neutrino mixings and 
leptonic CP violation," Physical Review D, vol. 68, no. 11, Article ID 115008, 2003.

[98] S. Kaneko, M. Katsumata, and M. Tanimoto, "Leptogenesis in neutrino textures with two zeros," Journal of High Energy Physics, vol. 2003, article 025, 2003.

[99] M. Hirsch, A. S. Joshipura, S. Kaneko, and J. W. F. Valle, "Predictive flavor symmetries of the neutrino mass matrix," Physical Review Letters, vol. 99, no. 15, Article ID 151802, 2007.

[100] S. Dev, S. Kumar, S. Verma, and S. Gupta, "Phenomenological implications of a class of neutrino mass matrices," Nuclear Physics B, vol. 784, no. 1-2, pp. 103-117, 2007.

[101] S. Dev, S. Kumar, S. Verma, and S. Gupta, "Phenomenology of two-texture zero neutrino mass matrices," Physical Review D, vol. 76, no. 1, Article ID 013002, 15 pages, 2007.

[102] I. de Medeiros, S. F. Varzielas, and G. G. Ross, “Tri-bimaximal neutrino mixing from discrete subgroups of $\mathrm{SU}(3)$ and $\mathrm{SO}(3)$ family symmetry," Physics Letters B, vol. 644, no. 2-3, pp. 153157, 2007.

[103] A. A. Aguilar-Arevalo, C. E. Anderson, S. J. Brice et al., "Event excess in the MiniBooNE search for $\bar{\nu}_{\mu} \rightarrow \bar{\nu}_{e}$ Oscillations," Physical Review Letters, vol. 105, Article ID 181801, 2010.

[104] A. Aguilar, L. B. Auerbach, R. L. Burman et al., "Evidence for neutrino oscillations from the observation of $\bar{\nu}_{e}$ appearance in a $\bar{\nu}_{\mu}$ beam," Physical Review D, vol. 64, Article ID 112007, 2001.

[105] Z. Hou, R. Keisler, L. Knox, M. Millea, and C. Reichardt, "How massless neutrinos affect the cosmic microwave background damping tail," Physical Review D, vol. 87, no. 8, Article ID 083008, 11 pages, 2013.

[106] M. Lavedor and C. Guinti, "Phenomenology of neutrino oscillations and mixing," Acta Physica Polonica B, vol. 44, no. 11, pp. 2323-2330, 2013.

[107] S. M. Bilenky, S. Pascoli, and S. Petcov, "Majorana neutrinos, neutrino mass spectrum, CP violation, and neutrinoless double $\beta$ decay. II. Mixing of four neutrinos," Physical Review D, vol. 64, Article ID 113003, 2001.

[108] A. S. Riis and S. Hannestad, "Detecting sterile neutrinos with KATRIN like experiments," Journal of Cosmology and Astroparticle Physics, vol. 2011, article 011, 2011.

[109] J. A. Barrett and J. Formaggio, "Resolving the reactor neutrino anomaly with the KATRIN neutrino experiment," Physics Letters $B$, vol. 706, no. 1, pp. 68-71, 2011. 

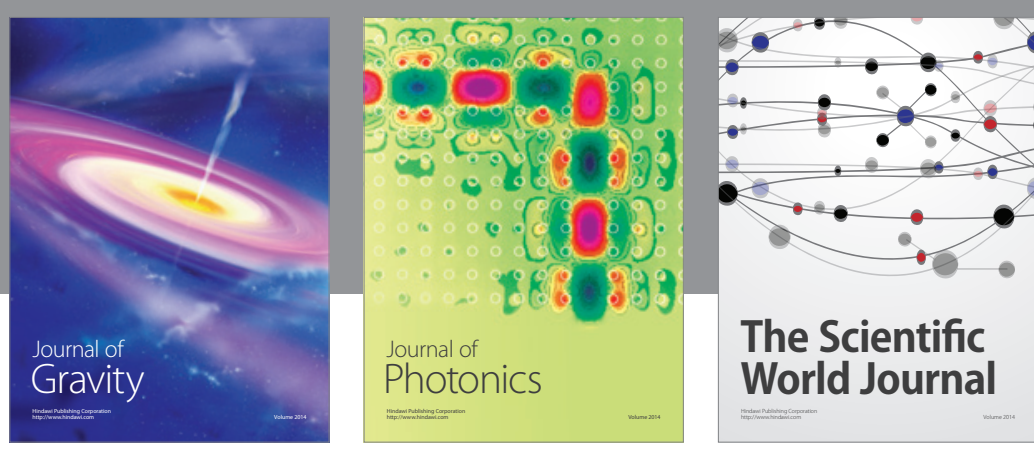

The Scientific World Journal
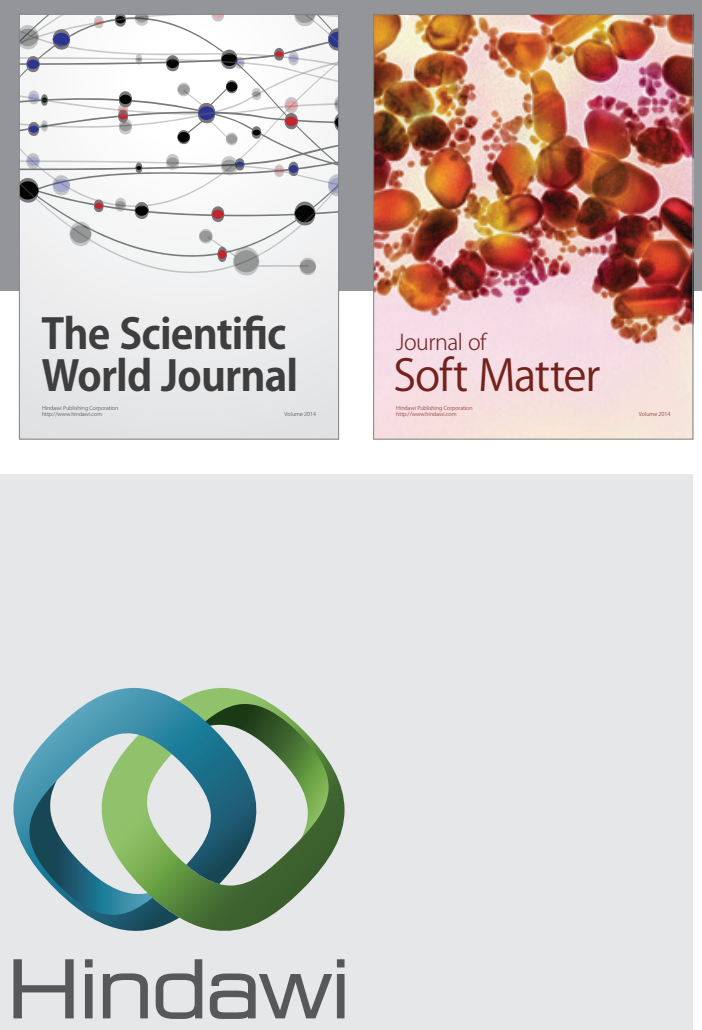

Submit your manuscripts at

http://www.hindawi.com

nternational Journal of

Statistical Mechanics
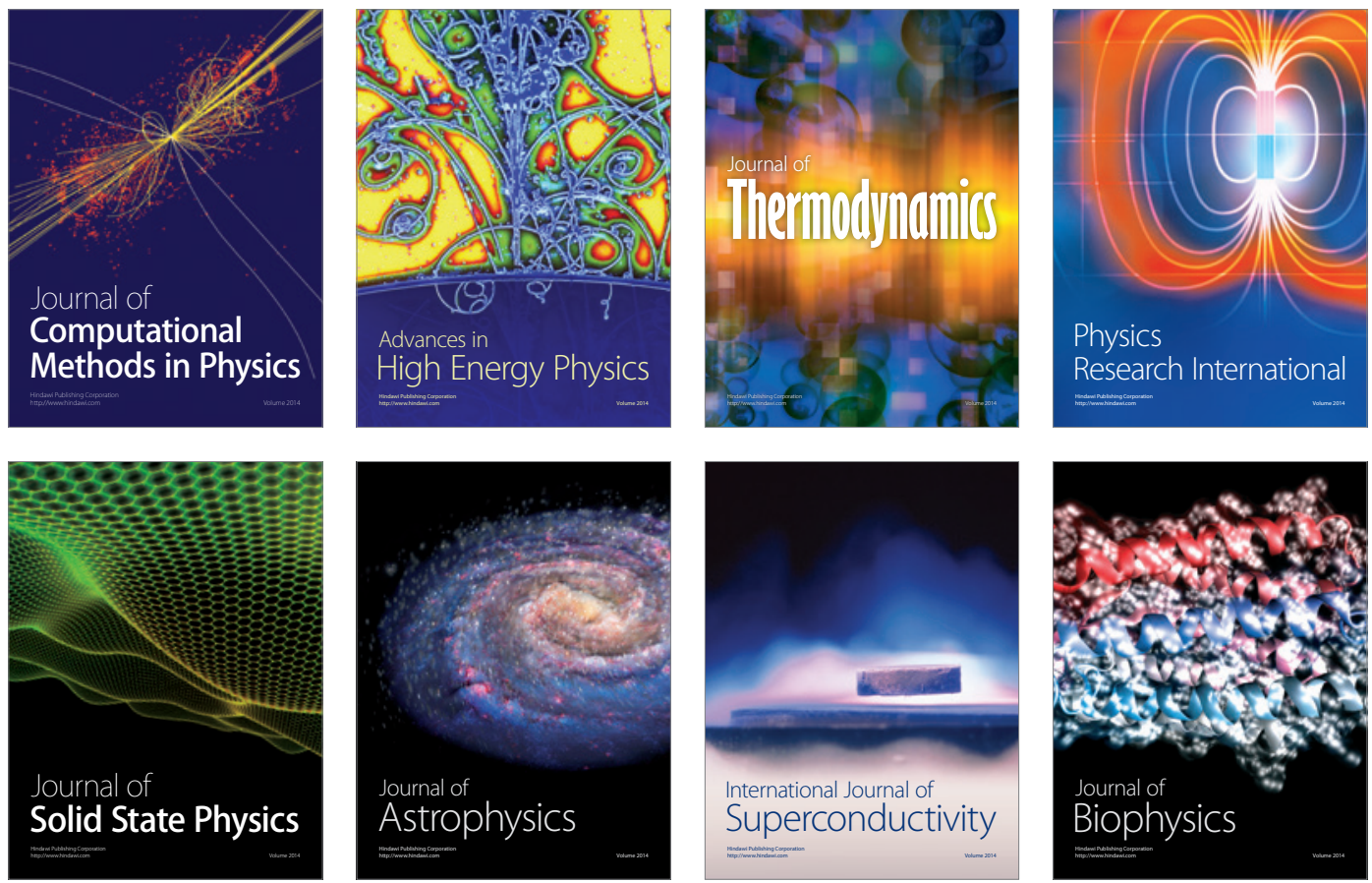
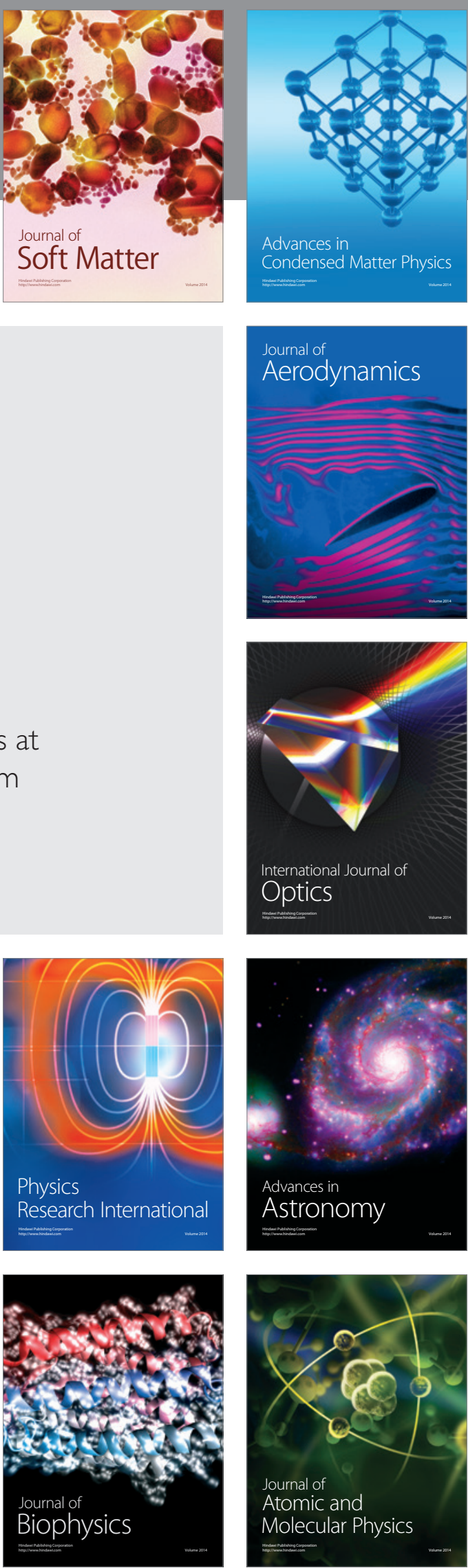